\title{
IP traceback through (authenticated) deterministic flow marking: an empirical evaluation
}

\author{
Vahid Aghaei-Foroushani* and A Nur Zincir-Heywood
}

\begin{abstract}
In this paper, we present a novel approach to IP traceback - deterministic flow marking (DFM). We evaluate this novel approach against two well-known IP traceback schemes. These are the probabilistic packet marking (PPM) and the deterministic packet marking (DPM) techniques. In order to do so, we analyzed these techniques in detail in terms of their performances and feasibilities on five Internet traces. These traces consist of Darpa 1999 traffic traces, CAIDA October 2012 traffic traces, MAWI December 2012 traffic traces, and Dal2010 traffic traces. We have employed 16 performance metrics to evaluate their performances. The empirical results show that the novel DFM technique can reduce the number of marked packets by $91 \%$ compared to the DPM, while achieving the same or better performance in terms of its ability to trace back the attack. Additionally, DFM provides an optional authentication so that a compromised router cannot forge markings of other uncompromised routers. Unlike PPM and DPM that trace the attack up to the ingress interface of the edge router close to the attacker, DFM allows the victim to trace the origin of incorrect or spoofed source addresses up to the attacker node, even if the attack has been originated from a network behind a network address translation (NAT) server. Our results show that DFM can reach up to approximately 99\% traceback rate with no false positives.
\end{abstract}

Keywords: Flow base IP traceback; DDoS attacks; Deterministic flow marking; Authenticated flow marking; Security

\section{Introduction}

In recent years, much attention has been paid for securing the Internet infrastructure that has become a universal medium for a broad range of communications. Several security approaches have been proposed for securing this infrastructure. The specific security issue, which is the main focus of this study, is anonymous attacks. Due to the trusting nature of the IP protocol, which originally did not include security as a design principle, the source IP address of a packet is not authenticated. Attackers are usually interested in hiding their identity with fake addresses. (Distributed) Denial of Service ((D)DoS) attacks are an example of anonymous attacks where currently there is no obvious way to prevent or trace them. While preventing all attacks on the Internet is far from reality, at least a mechanism of identifying the source(s) of the attack is needed

*Correspondence: vahid@cs.dal.ca

Faculty of Computer Science, Dalhousie University, Halifax, Nova Scotia B3H IW5, Canada in a situation when prevention fails. This is the reason for designing IP traceback techniques. Traceback is a name given to any method for reliably determining the origin of traffic on the network.

To the best of our knowledge, the state-of-the-art traceback methods in the literature are able to detect only up to the autonomous system (AS) level or at best, the edge router of the attacker network, because proxies and network address translations (NATs) make it difficult to differentiate activities from distinct hosts. In these cases, to defend against attacks, the victim filters out all traffic belonging to the attacker network after identifying the origin of the attack.

Consider a scenario where a computer in a large network, such as a university, starts (D)DoS attack by spoofing its IP address against a public server (victim). At best, if an IP traceback technique has been utilized in both the university network and the victim side, the victim would be able to identify that the attacker's network is the university network. When the victim starts filtering

\section{照 Springer}


out any traffic from this network, many computers at the university network that have not been involved in the attack will also be filtered out.

In general, there are two ways for routing the network traffic from a local network to the Internet:

1. Valid IP addresses are assigned to the local nodes. The edge router only routes the traffic to and from the Internet. We call these networks as 'valid networks'.

2. An invalid IP address is assigned to each node in the local network. In addition to routing, the edge router should do the network address translation. We call these networks as 'invalid networks'.

Table 1 shows the differences of these two networks from the perspective of a destination node outside of these networks. As can be seen in this table, only in a situation when a computer does not send spoofed source IP address packets from a valid network that the destination is able to distinguish the exact source of this traffic. In the other three situations, the destination is not able to identify the source node, i.e., the origin of the traffic. Therefore, in the case of attack traffic coming from such networks, the destination (in this case the victim) has to filter out all the traffic flows originating from the attacker's network.

In this research, we aim to deal with such a problem and propose an IP traceback method to be able to identify the actual attacking machine as accurate as possible in a given network. First of all, we present a brief survey of two promising schemes for tracing cyber-attacks, the wellknown probabilistic packet marking (PPM) and the deterministic packet marking (DPM) approaches. Then we present a novel approach to IP traceback - deterministic flow marking (DFM). We explore the PPM and DPM in detail and then by investigating the DFM, we analyze the advantages and disadvantages of all three approaches in depth in terms of practicality and feasibility so that the shortcomings of each scheme are highlighted. We employ five different Internet traces (data sets) including Darpa 1999 attack and attack free, Cooperative Association for Internet Data Analysis (CAIDA) Internet traces October 2012, measurement and analysis of WIDE-area Internet (MAWI) December 2012, and Dalhousie 2010 data sets, and use 16 metrics to evaluate the performance of disparate traceback schemes. The metrics employed in this work are the following: the computational overhead, the memory overhead, the bandwidth overhead, the traceback rate, the false-positive rate, mark spoofing by attackers or subverted routers in the attack path, the awareness of the attack path length, the network map and the routing in advance, the number of required packets for traceback, the percentage of marked packets, Internet service providers (ISP) involvement, the ability to handle fragmentation, the ability to handle major DDoS attacks, and the maximum traceback ability.

The main characteristics of our research that distinguishes it from other methods include the following:

1. We propose a generalized deterministic flow marking scheme, DFM, which is scalable to large distributed attacks. DFM outperforms PPM and DPM in that it can handle larger-scale DDoS attacks because the maximum number of concurrent attackers in PPM and DPM is limited, whereas there is no such limitation in DFM.

2. Although deterministic IP traceback methods have higher traceback accuracy in comparison to probabilistic marking approaches, this accuracy is achieved by marking all the packets in the network. Deterministic methods need to process every packet and obviously, they incur more processing overhead [1]. In our proposed DFM technique, we aim to minimize this overhead. To achieve this, given that all packets in a flow belong to the same source, we mark every flow, instead of every packet. This leads us to have both advantages of high traceback accuracy of deterministic methods and low processing overhead of probabilistic approaches simultaneously. Our experimental results show that the proposed DFM method has approximately $99 \%$ traceback rate with $0 \%$ false-positive rate, while it may reduce the number of required packets to be marked for tracing back by $90 \%$.

3. Most of the traceback methods assume that the marking information remains unchanged for as long as the packet traverses the network. Unfortunately, such an assumption is not realistic given the issue of mark spoofing by forged routers. DFM totally eliminates the threat of mark spoofing, not only if spoofed marking is inscribed by the attacker, but also if it is incurred by the compromised routers in the attack path. We show that this can be accomplished by using optional authenticated flow marking.

Table 1 The differences between valid and invalid networks

\begin{tabular}{lll}
\hline Network type & Sending legitimate flows & Sending attack flows \\
\hline Valid & Destination is able to identify the origin of traffic & If an attacker spoofs its source IP address, the destination (victim) \\
& cannot identify the origin of the attack traffic \\
Invalid & Destination cannot identify the origin of traffic & The destination (victim) cannot identify the origin of the attack traffic \\
\hline
\end{tabular}


4. Finally, unlike DPM that traces the attack up to the ingress interface of the edge router close to the attacker, DFM allows the victim to trace the origin of the incorrect or the spoofed source addresses up to the attacker node, even if the attack has been originated from a network behind a NAT server.

The rest of this paper has the following structure: Section 2 summarizes the related work on IP traceback and various traceback schemes are classified from multiple aspects. Then, the actual schemes of PPM, DPM, our proposed DFM, and its optional authenticated flow marking feature are presented; and the implications and the challenges associated with each of them are discussed from the perspective of practicality and feasibility in Sections 3, 4, and 5, respectively. Finally, we provide a comprehensive comparison table for all schemes and present our conclusions in Section 6.

\section{Literature review on IP traceback}

So far, many traceback approaches have been proposed. According to [1] and [2], we classify existing approaches from multiple viewpoints. Three aspects are selected to classify existing traceback schemes into several categories. They include the basic principle, processing mode, and location.

According to classification by the basic principle, most of the existing traceback methods are categorized into logging and marking groups. In logging methods, the routers keep some specific information of traveling packets [3]. For example, Snoeren et al. [4] have suggested generating a fingerprint of the packet, based upon the invariant portions of the packet (source, destination, etc.) and the first 8 bytes of the payload. During the traceback, the routers can verify if a suspicious packet has been forwarded or not. Further improvement in terms of logging only a small portion of each traveling packet at the transient routers have been proposed in [5]. One of the major problems of the logging method is the requirement for high amount of memory and CPU usage on the routers in the attack paths [6]. In marking methods, some or all routers in an attack path send specific information along with traveling packets. The destination may use this information to trace the attacker even if the source IP has been spoofed. This information could be either embedded in the packet's IP header or sent by generating new packets and consume extra bandwidth [7-10]. In particular, Savage et al. [11] have described a technique for tracing anonymous packet flooding attacks on the Internet towards their source. This traceback can be performed after an attack is identified. While each marked packet represents only a sample of the path it has traversed, by combining a modest number of such packets, a victim can reconstruct the entire attack path. Dean et al. [12] have presented a scheme for providing traceback data by having routers embedding specific information into packets randomly. This is similar to the technique used by Savage et al. [11], with the major difference being that it is based on algebraic techniques. On the other hand, Song et al. [13] present two new IP marking techniques to solve the IP traceback problem: the advanced marking scheme and the authenticated marking scheme. The authenticated marking scheme supports authentication of routers' markings. This prevents a compromised router from forging other uncompromised routers' markings. Doeppner et al. [14] identified the source of Denial of Service attacks, provided that a significant percentage of packets are sent from one subnet. In this method, each router marks its own IP address to the traveling packet with a determinable probability. Moreover, Tseng et al. [15] have proposed a modification to PPM [11] to ensure that the probability of receiving the mark is equal to the original marking probability. Yaar et al. [16] have proposed a method of encoding path identification by marking packets with path fingerprints. They have also another research [17] based on the PPM [11] with further improvements such as 1-bit distance. Victims can identify attack paths after receiving tens of packets encoding. It detects the distance of the attacker by changing the time to live (TTL) field and storing 1 bit in the IP header. Goodrich et al. [18] have proposed to use relatively large randomized messages to encode router information. The main idea is to have each router fragment its message into several words and include a large checksum cord on the entire message randomly in the reusable bits of such a word fragment. Instead of the recovery of the full paths, Belenky et al. [19] and [20], proposed to only record the IP addresses of ingress edge routers. Their scheme, DPM, is simple and easy to implement, and has a little overhead on routers and the victim. This scheme has low processing and memory overhead at the victim machines and edge routers. Additionally, DFM provides an optional authentication so that a compromised router cannot forge markings of other uncompromised routers. Yang et al. [21] take advantage of both marking and logging methods and combines both approaches at routers in an attack path. Most marking methods use 16 bits of identification field such as in [11,13,15-17]. However, some other works propose to use 17 bits (identification field and reserved flag) [20,22], 25 bits (identification and type of service (TOS) fields plus reserved flag) $[12,18,23]$, or 32 bits (identification field, flag, and fragment offset) $[9,21]$.

From the perspective of the classification based on the processing mode, traceback schemes may be categorized into two groups: deterministic and probabilistic. In deterministic methods, regardless of the marking or logging, every packet should be processed at both the source and 
the destination side. In comparison to the probabilistic methods, these methods require more processing overhead but their advantage is providing higher accuracy. An example of probabilistic logging is proposed by Snoeren et al. [4], which was discussed earlier. There are some researches on deterministic marking as well. For example, the suggested idea by Belenky and Ansari [20] is to store, with random probability of 0.5 , the upper or the lower half of the IP address of the ingress interface into the fragment ID field of the packet, and then set a reserve bit indicating which portion of the address is contained in the fragment field. The proposed method by Rayanchu and Barua [22] is similar to Belenky and Ansari [20], but the difference is that they do not embed the IP address in the IP header; instead they only embed the hash of the edge router's IP address. Most of the recent traceback methods are probabilistic. While the required bandwidth and processing time in these methods are less than the ones required by the deterministic methods, the complexity for reconstruction at the destination side is more. Some wellknown examples of probabilistic methods are PPM [11] and many of its variants [15,17], ATA [12], iTrace [7], and others such as in $[9,13,14,16-18]$.

From the perspective of the classification by locations, existing traceback methods are divided into two types: those that send traceback information by the edge routers closest to the source (source group) and in the network by some or all routers in the attack path (network group), respectively. Most of the current traceback methods belong to the network group [11-14]. The purpose of these methods is to identify the attack path entirely or partially $[9,15-17]$. The drawbacks of these methods are the involvement of the routers along the paths and the cost of their processing times and memories for this purpose $[4,21,23]$. While the goal of source group methods is to identify the attack source, they do not identify the attack path $[18,20,22]$.

Furthermore, the proposed methods in $[9,23,24]$ trace up to the autonomous system (AS) level, while the other aforementioned works trace up to the edge router of the attack source. Song et al. [13] and Goodrich et al. [18] have proposed authentication marking methods, while the other aforementioned works send their marking information in clear text that are susceptible for mark changing in the case of existing compromised routers in the network path. Wang et al. [25] proposed a framework for 'Sleepy Watermark Traceback (SWT)' (i.e., watermarking and tracing packets to the attacker's source IP address, only if the IDS subsystem has determined that there is an attack in progress). This technique is based on traffic timing and is quite different from the ones mentioned above in that it injects non-displayable contents into packets. SWT has the following assumptions: (1) Intrusions are interactive and bidirectional. This paper refers to intrusions as those attacks aiming to gain unauthorized access, rather than Denial of Service attacks, so it is not able to trace back Denial of Service attacks that are bidirectional, and attempts to block access to the targeted server by consuming computing resources on the server and by consuming all of the bandwidth of the network connecting the server to the Internet. (2) Routers are trustworthy. (3) There is no link-to-link encryption. So this method is vulnerable to encrypted traffic.

In our previous work, we proposed the DFM approach which allows the victim to trace back the origin of an incorrect or spoofed source IP address up to the attacker node, even if the attack has been originated from a network behind a NAT server [26]. DFM is a deterministic approach, which marks every flow (in contrast with making every packet) and performs on the edge router near the source of traffic. We have shown that using DFM may reduce as many as $90 \%$ of marked packets on average required for tracing attacks with no false positives, while it eliminates the spoofed marking embedded by the attacker as well as compromised routers in the attack path in [27].

\section{Probabilistic packet marking}

In this section, we describe the probabilistic packet marking method called PPM. Based on various IP traceback approaches described in Section 2, PPM falls into the following categories: basic principle - marking, processing modes - probabilistic, and location - network group.

This approach is based on the idea that all routers in the attack path select the packets that pass through them randomly, with a constant probability, and then mark the selected packets by their own IP address (i.e., write a portion of their own IP address in the packet IP header). Once the victim gets a large amount of marked packets, it can reconstruct the attack path, even if the IP addresses of the packets have been spoofed. This approach, Figure 1, had been introduced by [28] and later has been improved by [8]. Assume that there are $d$ routers in an attack path and the marking probability of each of these routers is a constant number $p$. The optimal value for $p$ is $1 / d$. However, from the viewpoint of victim, the marking probability of router $R^{i}(1=>i<=d)$ is $p(1-p)^{d-i}$ that is different than $P[15,29]$. It is because subsequent routers may override (re-mark) the packets that have been marked by the previous routers. In other words, the routers that are further away from the victim are, more likely to be overridden by the subsequence routers. Thus, the closest router to the victim has the highest chance to deliver its marks in the attack path.

\subsection{Mark decoding by destination}

In this approach, there are two fields, addr and dist, that play the main role in the packet marking. Both of these 


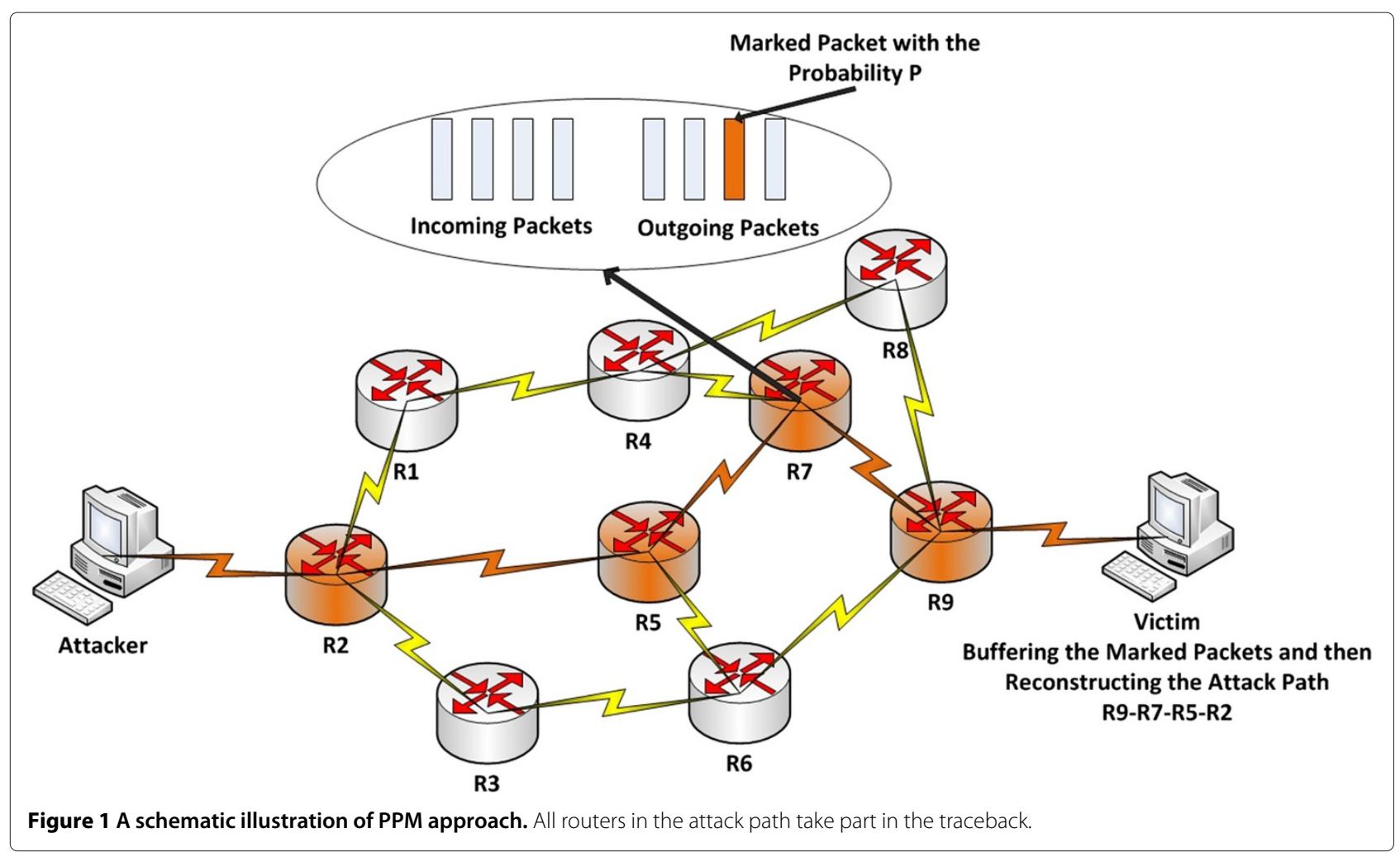

fields have been embedded in the identification field of packet IP header. Once a router decides to mark a packet (i.e., this decision is independent of other routers), it writes its own IP address to the addr field and zero in dist field. Otherwise, if the router gets a packet that its dist field is zero, it indicates that this packet has been marked by the previous router. In this case, the router would XOR its own IP address with the addr field of the marked packet and would override the result into the addr field again. Finally, if the router does not mark the packet, it always adds one to the dist field.

Figure 2 shows the marking process in an attack path with three routers $R 1, R 2$, and $R 3$. In the $R 1$ router, there are two cases. The box on the left shows the case that the $R 1$ marks the packets (i.e., it writes its own IP address in addr field and set the dist field to zero), and the box on the right shows the unmarking case by the $R 1$ router (i.e., it just adds one to the dist field). In the $R 2$ router, there are four cases, two boxes on the left show a situation where the $R 1$ router has previously marked the packets. Of these two boxes, the box on the left shows the case that the $R 2$ router marks the packet again (i.e., it writes its own IP address in the addr field and set the dist field to zero), and the box on the right shows the case that the $R 2$ router does not mark the packet (i.e., it XOR its own IP address with the addr field, overrides the result into the addr field, and add one to the dist field). On the other hand, two boxes on the right show a situation where the $R 1$ router has not previously marked the packets. Of these two boxes, the box on the left shows the case that the $R 2$ router marks the packet (i.e., it writes its own IP address in the addr field and set the dist field to zero), and the box on the right shows the case that $R 2$ router does not mark the packet (i.e., it just adds one to the dist field). Using the same process for the $R 3$ router, the victim would get eight results. However, some of these eight marking results are similar. For example, boxes number 1, 3, 5, and 7 have the same marking information. By eliminating the duplicate results, four non-repetitive cases remain for the victim.

For the path reconstruction, first of all the victim locates the closest router to itself (i.e., $R 3$ in Figure 2) by looking for the packet where the dist field is equal to zero. Secondly, because $R 3 \oplus(R 2 \oplus R 3)=R 2$, the victim can locate the $R 2$ router by looking for the packet where the dist field is equal to 1 , and XOR its addr field with the IP address of the $R 3$ router. The victim continues this process until locating the router that is the most far away.

\subsection{PPM analysis}

Although PPM has several good advantages such as zero bandwidth overhead (i.e., all marking information stored in the packet IP header), PPM has the following serious weaknesses in the face of DDoS attacks. 


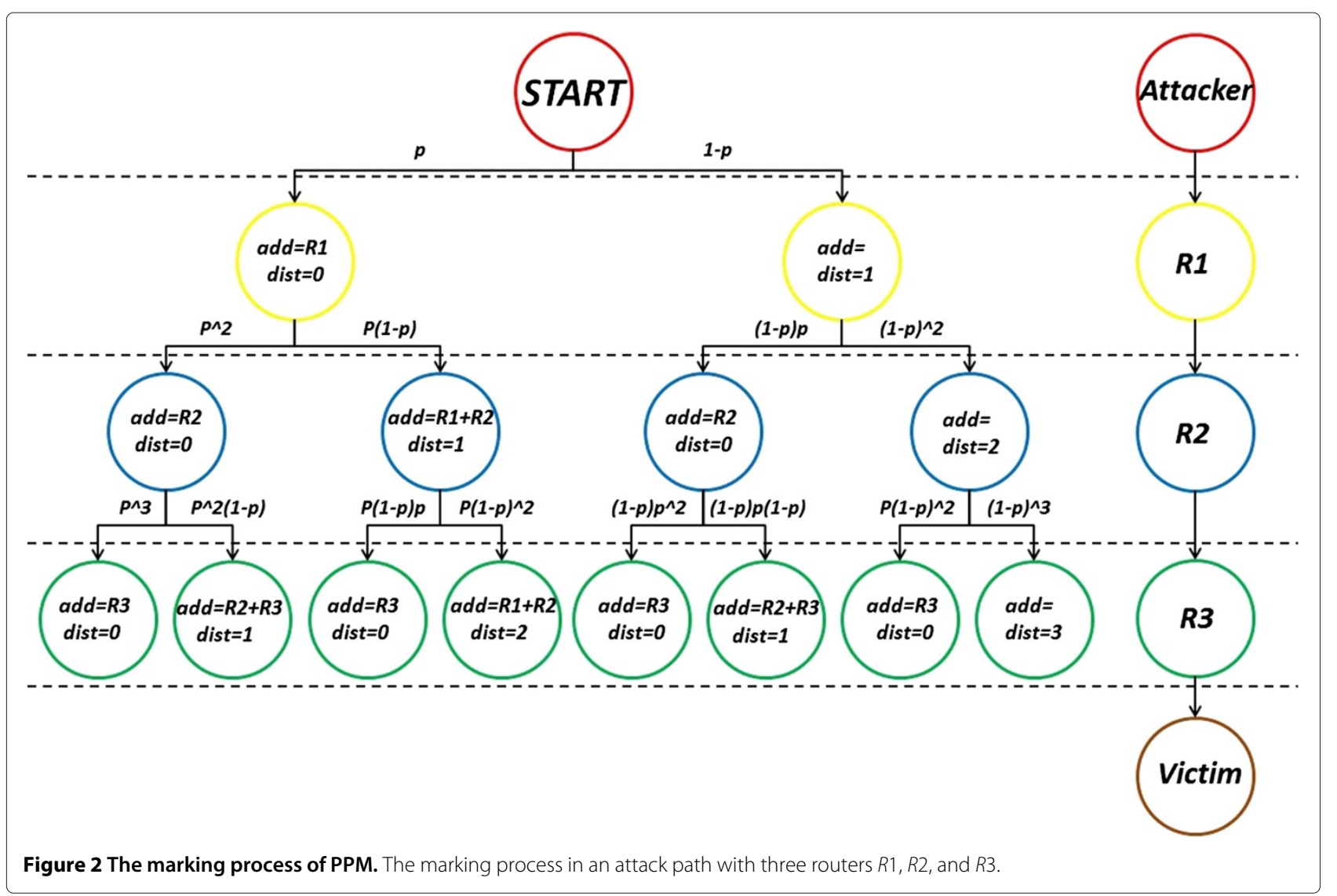

\subsubsection{Computational overhead}

For each packet, there is a computational overhead to decide if the packet should be marked or not. In addition, if the packet is marked, there are some more computational overheads such as preparing the marking information and upgrading the addr and the dist fields. However, in comparison to the computational overhead at the victim side for path reconstruction, the computational overhead of the routers in the attack path is negligible. Authors in [13] show that when there are 25 concurrent attacks to a victim, path reconstruction may take several days with thousands of false positives, while the current DDoS attackers may orchestrate thousands of attack zombies at the same time. In this situation, the victim may never reconstruct the attack path.

\subsubsection{Memory overhead}

The memory overhead on routers is highly undesirable because it reduces the network performance and requires hardware upgrade. Since the marking process on routers does not store anything, the router's memory overhead in PPM algorithms is negligible. But on the victim side, a large memory structure for the attack path reconstruction process is required. The victim should store millions of records in the data structure, and then search on it, to reconstruct the attack path. However, memory overhead on the victim machine is more tolerable than the one on the routers.

\subsubsection{False positives}

PPM has a high false-positive rate in the face of DDoS attacks. This problem is originated from the basis of the reconstruction algorithm. In this case, the victim should perform two processes; first of all, it should gain the IP addresses of all routers in the attack path and secondly, using the router's IP addresses, reconstructs the attack path. In PPM, eight packets marked by the same router need to be identified and combined to resume the IP address of that router [9]. Since there is no more sign in the dist field, in a situation when there are several attack paths, it is difficult for the victim to identify which marked packets belong to which router because there are lots of routers in the same distance to the victim. This issue may prevent the attack path reconstruction process.

\subsubsection{Mark spoofing by attackers}

If the attacker is aware of the existing PPM marking process in the network, he/she may send fake marked packets 
to the victim. In this situation, the victim may not be able to reconstruct the attack path correctly because the victim cannot differentiate between the fake and the genuine marked packets.

\subsubsection{Mark spoofing by subverted routers}

There are two kinds of malfunctioning routers that may disrupt the traceback operation by the victim. First of all, the incorrect configured routers that participate in the PPM packet marking may confuse the path reconstruction process. Secondly, the compromised routers can prepare and send fake marked packets that can most likely prevent the victim to trace back to the attack source.

\subsubsection{Awareness of the attack path length in advance}

As described before, the optimal value of marking probability, $p$, is $1 / d$. However, once a router decides to mark a packet, it does not have any idea about its path length, $d$, so it cannot set the $p$ to the optimal value. Authors in [8] suggest to use the constant number 0.04 for $p$. However, if the victim is under several attacks with different attack path lengths, using the predetermined constant number for $p$ strongly reduces the efficiency of the path reconstruction process.

\subsubsection{Awareness of the network map and the routing in advance}

The PPM algorithm works based on an assumption that the victim should be aware of the network map and the routing in advance to be able to reconstruct the attack path using the IP addresses of the routers among the path extracted from the received marked packets. So the concern in this case is how to keep the victim updated about the network map and routing; otherwise, whenever a new router is added to the network, the path reconstruction process will not work correctly.

\subsubsection{The number of required packets for traceback}

In the first implementation of PPM algorithm, the victim requires thousands of packets to reconstruct an attack path [8]. Later, this has been improved to less than 1,000 packets by [13]. However, the number is still high and therefore is a serious drawback of the PPM algorithm.

\subsubsection{Fragmentation}

PPM uses ID field in the IP header of packets to embed marking information, which is generally used for fragmentation. If only a single packet of a fragmented datagram is marked, then the datagram reassembly will fail.

\subsubsection{ISP involvement}

The path reconstruction process needs to get marked packets from all routers among the attack path. To this end, the marking process should be activated on all routers in the network. However, what the Internet service providers (ISPs) need to do is limited to updating the router's IOS and enabling the PPM on the routers. Having said this, ISPs should do this on all routers, including either edge or backbone routers. Indeed, the involvement of all the routers is a major problem of using this method. Given that, in practice it may cross boundaries of ISPs and countries.

\subsection{Discussion}

So far, several variations of PPM have been proposed $[8,15,17]$. For example, to counter with 'awareness of the attack path length in advance' problem, there are some works $[9,23,24]$ to set $d$ as the number of AS, rather than the number of routers, between the current network and the victim. However, this solution cannot reconstruct the attack path accurately, which is the main goal of the PPM approach.

Song [13] has proposed an advanced and authenticated marking scheme for IP traceback. Their approach decreases the high computational overhead and falsepositive rate, as the number of the required marked packets for path reconstruction is less than 1,000 in their approach and partially covers the mark spoofing problem. However, their approach cannot resist against the compromised routers in the attack path, since a compromised router may be reconfigured to mark the packets incorrectly and still is authenticated by the victim. Unfortunately, there is still no approach to cover the problem of malfunctioned routers. Note that the computational overhead and the false-positive rate are in direct proportion; as the high computational cost increases, so does the false-positive rate.

One possible solution to counter with the fragmentation problem is to mark the fragmented packets with lower probability. Therefore, the fragmented datagrams have more chance to survive. However, this approach will definitely increase the number of required marked packets for path reconstruction.

As described earlier, as the number of hops between a router in the attack path and the victim increases, the mark information of that specific router is less likely to survive; so from the perspective of the victim, the farthermost router has the lowest chance to deliver its mark in the attack path. One solution to cancel this problem is to use variable marking probability for each router, based on the distance between the current router and the victim [30]. However, the hard part is how to find the number of hops between two ends.

Based on the above PPM specifications, PPM is a good solution for small-scale DoS attacks. However, most of the current attacks are large-scale DDoS attacks with hundreds or thousands of concurrent attack sources. In these cases, PPM is not a good choice. Therefore, researchers 
proposed another approach, called DPM [19], to overcome some of the problems of the PPM approach.

\section{Deterministic packet marking}

DPM is a well-known IP traceback approach and possesses several attractive features such as its ease of implementation and low computational and memory overhead on participating routers as well as the victim machines. Based on various IP traceback approaches described in Section 2, DFM falls into the following categories: basic principle - marking, processing modes - deterministic at packet level, and location - source group.

\subsection{DPM scheme}

The main goal of DPM, which was first proposed by Belenky and Ansari [19] and later was improved by [20], was to dominate on an obvious issue of PPM. The issue was that each packet in a datagram network is being routed individually so even if the sources and the destinations of the packets are the same, they may be routed in different paths. This feature of the packet networks may prevent the attack path reconstruction by victim, using the PPM algorithm. Since each packet may travel a different route from the same source to the same destination, the only address in the network path that is surely the same for all packets is the ingress interface IP address of the closest router to the source of packets. The main idea behind the DPM is that the ingress interface IP address of the closest router to the source of the packet is enough to find the attacker network. It should be noted here that in the current Internet network, the packet routing is mostly stable. However, there is still this potential to route the packets from different paths.

As it is shown in Figure 3, only the ingress interfaces of the edge router marks the packets, and the rest, including the backbone routers, are exempt. DPM uses 17 bits of the IP header, including 16 bits identification field and 1 bit reserved flag, to embed the marking information to every packet. The 32 bits ingress interface IP address is split into two segments, with 16 bits each: segment 0 - bits 0 through 15 and segment 1 - bits 16 through 31 . When a packet passes through an edge router, one segment is selected with equal probability and inserted in the identification field. The victim maintains a table matching the source addresses to the ingress addresses. When the victim gets both segments of an edge router, then it is able to reconstruct the whole ingress interface IP address of that router. One-bit reserved flag plays the rule of a sign for the victim to identify which part of the IP address is carried by the current packet.

DPM has two key features: First of all, DPM only marks the closest ingress edge router to the attacker, and secondly, DPM marks all packets at the ingress interface of the edge routers.
Although the basic DPM approach can handle DoS attacks, it has high false-positive rates under DDoS attacks. The reason behind this is that the victim associates segments of the ingress address with the source address of the attacker. However, it is a well-known fact that the source IP addresses may be spoofed. Under such attacks, there are at least two cases when the edge router IP address reconstruction may not be effective. Firstly, two or more hosts that have the same source IP address attack the victim and secondly, (D)DoS attackers simply change the source address field for every packet they send. In these cases, the basic DPM is unable to reconstruct any valid ingress addresses [19]. To solve this problem, they improved their basic DPM approach to use a hash function to produce digests or hash values of the ingress address [20]. They proposed that all packets belonging to the ingress interface of an edge router carry the same hash value. Using this hash value, the victim is able to match the correct mark information to form a valid ingress IP address. Therefore, the marking information is formed by three parts: a segment of ingress address $a$, the index of segment $d$, and digest of ingress address $k$. They claimed that the best trade-off for the size of each of these parameters are $a=4, d=3$, and $k=10$, all together 17 bits.

\subsection{DPM analysis}

To analyze the DPM, we have used the same evaluation metrics that we used to analyze the PPM as discussed in the succeeding sections.

\subsubsection{Computational overhead}

The CPU overhead of DPM is lower than the PPM approach because unlike PPM, in DPM only the closest edge router to the attacker is responsible for marking (not all routers in the attack path). Moreover, since DPM marks every packet, there is no need for a decision process for marking each packet. However, there are other computational overheads such as preparing marking information and upgrading marking fields. Having said this, in DPM, reconstructing the ingress interface IP address of the edge router is much simpler than the attack path reconstruction process of the PPM approach. Therefore, in the face of DDoS attacks, the victim is able to trace back to the edge router in real time, if DPM is in use. Furthermore, the hash values of the ingress address may be used as a guide to effectively prevent the combinatorial explosion problem of PPM.

\subsubsection{Memory overhead}

Like the PPM approach, the memory overhead on the routers is negligible. However, unlike PPM where the victim requires a large memory structure to store millions of records, in the DPM approach, the victim keeps a smaller 


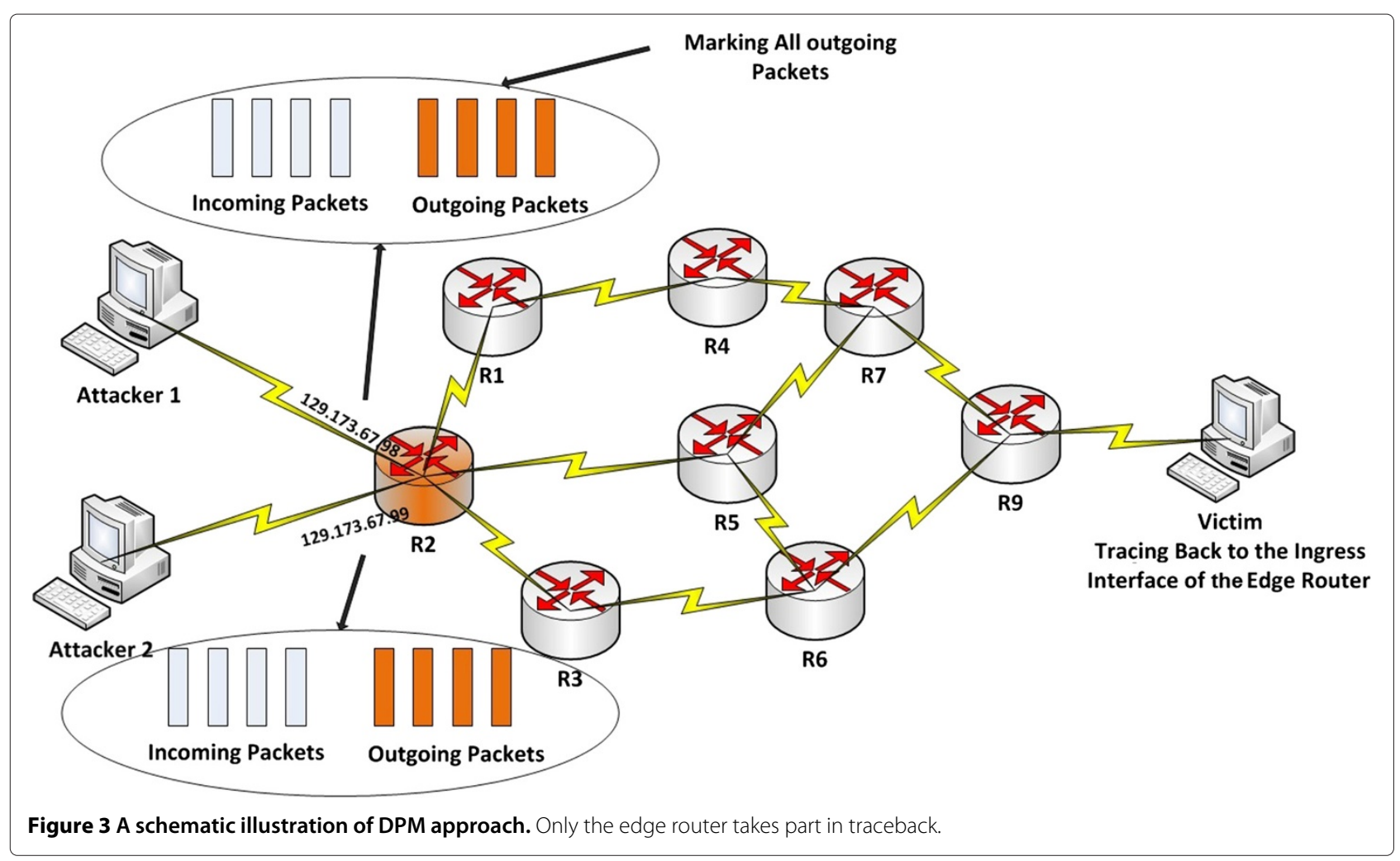

reconstruction table. It is because in PPM, the victim needs almost 1,000 packets to reconstruct an attack path, while in DPM, only $32 / a$ packets are required to reconstruct the ingress address (i.e., with the suggested $a=4$ [20], DPM requires only eight packets to trace back to the ingress interface address of the edge router close to the attacker).

\subsubsection{False positives}

As discussed earlier, basic DPM method has a significant limitation to deal with multiple attackers at the same time with the same source IP address. In this situation, the victim cannot recognize which marked fragment should be concatenated together to form a valid mark. This causes high false-positive rates. To counter this problem, they propose another method to use a hash function to produce hash values of the ingress interface, called the single-digest DPM technique, or to use a family of hash functions to produce multiple digests of an ingress address, called the multiple-digest DPM technique. In these techniques, hash values are sent along with marked bits to effectively prevent the combinatorial explosion problem. This modification to DPM guarantees the false-positive rate not to go over $1 \%$, if the number of concurrent attackers in a DDoS attack is not more than a limited number. For example, using 55 datagrams to be marked by the DPMenabled interface, the maximum number of simultaneous attackers that can be traced back with the false-positive rate not exceeding $1 \%$ in the single-digest DPM technique is 45 , and in the multiple-digest DPM technique is 2,296 [20].

\subsubsection{Mark spoofing by attackers}

In the DPM approach, each packet is marked when it enters the network. In this case, even if an attacker tries to spoof the mark, the spoofed mark will be overwritten with a correct mark. This automatically obviates the issue of the mark spoofing which PPM has to account for.

\subsubsection{Mark spoofing by subverted routers}

DPM assumes that a mark remains unchanged for as long as the packet traverses the network. As DPM does not have any mechanism to authenticate the packet marking, this assumption automatically increases the issue of mark spoofing by subverted routers in the attack path. Thus, in an untrusted network such as the Internet and in the case of a compromised router on the attack path, the marking information could be changed and the destination would be unable to identify the origin of the traffic.

\subsubsection{Awareness of the attack path length in advance}

As the process of ingress interface IP address reconstruction does not need the path length, awareness of the attack path length is not an issue in DPM. 


\subsubsection{Awareness of the network map and the routing in advance}

As the goal of DPM is not reconstructing the attack path, instead it reconstructs the ingress interface IP address of the edge router, so the awareness of the network map and the routing is not an issue.

\subsubsection{The number of required packets for traceback}

As discussed earlier, 32/a packets are required to reconstruct the ingress address. By the suggested $a=4$ [20], DPM requires eight packets to trace back to the ingress interface address of the edge router close to the attacker, where $a$ refers to the number of bits in a segment of the ingress address field.

\subsubsection{Fragmentation}

Like PPM, DPM uses the ID field in the IP header of the packets as well as 1-bit reserved flag to embed the marking information. If only a single packet of a fragmented datagram is marked, then the datagram reassembly will fail.

\subsubsection{ISP involvement}

In DPM, the involvement of the ISPs is very limited. Only the edge routers have to be upgraded to support the function of the deterministic packet marking. Unlike PPM, the other routers in the attack path and the network backbone do not need to be responsible for any function of the DPM traceback process.

\subsection{Discussion}

In summary, DPM mitigates some of the problems of the PPM. These are the following: its CPU and memory burden are far less; it improves the false-positive rate; limits sending the mark spoofed packets by the attackers; does not require for awareness of the attack path length, the network map, and the routing in advance; decreases the number of the required packets for the traceback from almost 1,000 packets to 8 packets; and the involvement of the ISPs is limited only to the edge routers. However, DPM has still some problems such as the following:

- To keep the false-positive rate not exceeding $1 \%$, DPM cannot scale under heavy DDoS attacks as discussed above [20].

- DPM is able to trace back up to the ingress interface of the edge router close to the attacker, not the exact attacker node.

- Although DPM has higher traceback accuracy in comparison to the probabilistic marking approaches, this accuracy is achieved by marking all the packets in the network.

- DPM assumes that the marking information remains unchanged for as long as the packet traverses the network. Unfortunately, such an assumption is not realistic given the issue of mark spoofing by forged routers.

The aforementioned four problems were our motivation for proposing the DFM approach [26,27].

\section{Deterministic flow marking}

In this section, we describe our proposed marking method called DFM. Based on various IP traceback approaches described in Section 2, DFM falls into the following categories: basic principle - marking, processing modes deterministic at flow level, and location - source group. The following are the assumptions of the DFM approach:

- Each node in a local network may change its IP address.

- Media access control (MAC) filtering is enabled in the edge router. However, the attacker may change its MAC address.

- DFM is not an intrusion detection or intrusion prevention system. It is a traceback system, which could work with the aforementioned systems to trace back to the source of the traffic that network managers or security engineers are interested in.

\subsection{Identifiers}

DFM uses three identifiers to mark a flow in order to trace up to the attacker node. These three identifiers are as follows:

1. The IP address of the egress interface of the edge router (32 bits): The edge router is the closest router to the attacker node with at least one valid assigned IP address to its egress interface. Some previous researches on IP traceback such as [20] and [31] have proposed to use the ingress interface IP address of the first router in the attack path as an identifier for traceback. However, since DFM should be able to trace up to the attacker node even if the attacker is behind a NAT, the ingress interface IP address will be useless in this case. Since the ingress interface IP address is invalid, the victim is unable to trace the source edge router by an invalid IP address.

2. Network interface identification (NI-ID) (12 bits): This is an identifier assigned to each interface of either the MAC address of a network interface on the edge router or the virtual local area network (VLAN) ID of a virtual interface if the edge router uses VLAN interfaces. The NI-ID specifies which subnet a traffic flow comes from. Currently, a router can equip 802.1Q VLAN interfaces, which means that multiple virtual interfaces can be used on one physical network interface. Although VLAN interfaces, which share one physical interface, have the same MAC address, each VLAN interface has a unique VLAN ID 
so that a router distinguishes VLAN interfaces by their VLAN IDs. If a network interface is a VLAN interface, a NI-ID is assigned to its VLAN ID instead of the MAC address shared with another VLAN interface. A 12-bit NI-ID, expressed in the range from 0 to 4,095, is sufficient to represent all possible network interfaces and VLANs on an edge router. An edge router keeps an NI-ID table and numbers the interfaces from 0 to 4,095. Each NI-ID table entry consists of an NI-ID and the MAC address of a network interface card. If VLAN interfaces are used, then the entry in the NI-ID table consists of an NI-ID and a VLAN ID. Table 2 shows the NI-ID table of an edge router which uses VLAN interfaces on a physical interface whose MAC address is ' $\mathrm{C}$ '.

3. Node-ID (16 bits): An identifier assigned to each source MAC address observed from incoming traffic from local networks. Each MAC has a unique Node-ID. Representing Node-ID with 16 bits seems to be sufficient as it makes possible to address all nodes on a LAN connected to each interface of the edge router, even if each LAN is as big as a class B network (the maximum number of nodes in a class $B$ network is $2^{16}-2$ ). An edge router keeps multiple Node-ID tables, a table for each NI-ID, and numbers the source MAC addresses of the observed incoming traffic from 0 to 65,535. An entry in the Node-ID table is composed of a NI-ID, source MAC addresses of the observed incoming traffic, and a Node-ID.

Table 3 shows two examples of a Node-ID table.

Marking each flow by a combination of the IP address of the egress interface (32 bits) + NI-ID (12 bits) + NodeID (16 bits) $=60$ bits identification data distinguishes the traffic of a particular node from the other nodes.

The definition of a flow is accepted as a unidirectional sequence of packets between two endpoints that have a flow ID in common with no more than a specific interpacket delay time. Flow ID is the five-tuple information including the source IP address, the destination IP address, the L4 protocol type (TCP/UDP), the source port number, and the destination port number. While this definition is able to define Transmission Control Protocol (TCP) and User Datagram Protocol (UDP) flows, it

Table 2 An example of a NI-ID table

\begin{tabular}{lcc}
\hline NIIID & $\begin{array}{c}\text { MAC address of the connected interface } \\
\text { of the edge router to the local network }\end{array}$ & VLAN ID \\
\hline 1 & A & \\
2 & B & 101 \\
3 & C & 102 \\
4 & C & \\
\hline
\end{tabular}

Table 3 Two examples of Node-ID table for NI-ID 1 and 2

\begin{tabular}{ccc}
\hline NI-ID & Node-ID & Source MAC addresses of incoming packets \\
\hline 1 & 1 & F \\
& 2 & E \\
3 & H \\
& 4 & K \\
& 1 & B \\
& 2 & S \\
& 3 & $\mathrm{Q}$ \\
\hline
\end{tabular}

is unable to define Internet Control Message Protocol (ICMP) flows, as ICMP does not use a port number to establish a session. For a good traceback method, it is very important to trace ICMP flows as well because some (D)DoS attacks employ ICMP flooding attack. To this end, we define an ICMP flow as a unidirectional sequence of ICMP packets between two networks that have the six-tuples as source IP address, destination IP address, L4 protocol type (ICMP), ICMP type, ICMP code, and ICMP ID in common with no more than a specific interpacket delay time. As used in [32], we define $600 \mathrm{~ms}$ for interpacket time delay to terminate a flow.

\subsection{Mark encoding by the edge router}

Figure 4 describes the marking procedure of our proposed traceback method. A 60-bit identification data needs to be passed to the destination for each flow. The identification data is divided into $K$ fragments. Therefore, the mark contains $M=60 / \mathrm{K}$ bits of the identification data and $S=\log _{2} \mathrm{~K}$ bits required to identify a fragment. We also take advantage of one flag bit to identify marked and unmarked packets in a flow. In the experimental results section, we describe how to store the marked bits in some IP header fields that are used rarely.

Figure 5 depicts our choice for partitioning the 60 bits in the first $K$ packets of each flow. The first $K$ packets of every flow carry the mark fragments including $M$ bits for identification data fragment, $S$ offset bits to represent $2 S$ possible fragments and one flag bit that should be set to ' 1 ' for the marked packets and ' 0 ' for the rest.

\subsection{Mark decoding by destination}

Figure 6 describes the mark decoding procedure. Each destination maintains a table matching the flow ID and $K$ possible mark fragments. We call this table the 'reconstruction table' (Table 4). As described before, the flow ID of TCP and UDP flows is defined by five-tuples while the flow ID of ICMP flows is defined by six-tuples. When a packet belonging to an unseen flow arrives at the destination, the destination creates a new table entry in the reconstruction table. Then, it extracts the marking bits of this flow from the marked packets, identified by one bit 


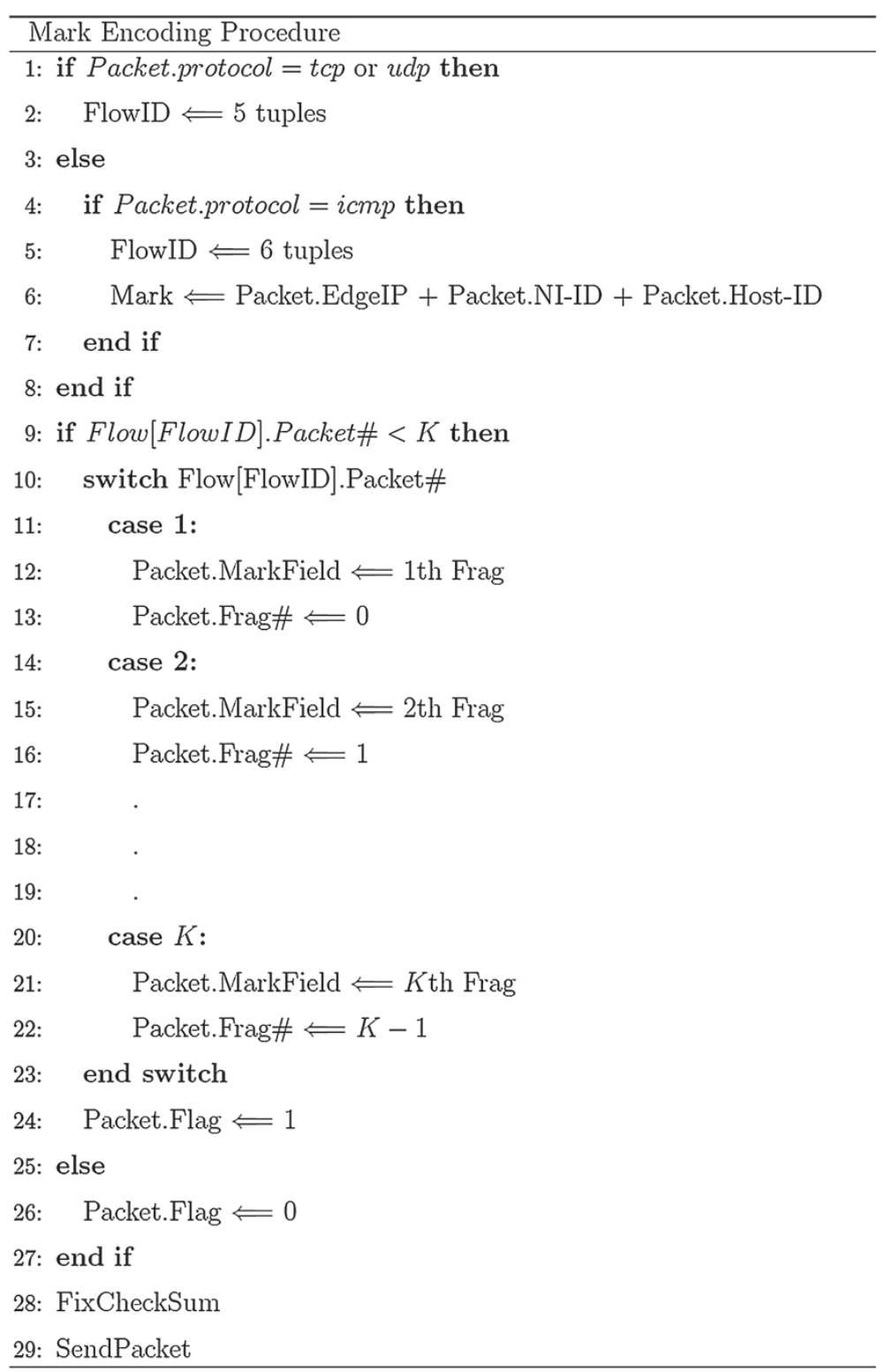

Figure 4 Mark encoding procedure.

flag, and writes them in the corresponding fields (Table 4). After all fragments corresponding to a flow reach the destination, the source node for the given flow becomes recognizable to the destination. Using DFM, the destination is able to distinguish the traffic of different nodes behind an edge router. As a result, when abnormal traffic is observed, the destination can filter the traffic of each node.

\subsection{Discussion}

As we discussed earlier, some previous traceback researches use hash functions and send the hash values along with the marked packets to counter the concurrent attacks. In particular, Belenky and Ansari [20] propose to insert the ingress interface IP address into outgoing packets by the first router of the attack path. Because the 32 bits that compose an IP address do not fit into the available marking space, they propose to split the router IP address into $K$ fragments. After receiving all $K$ address fragments, the victim can recover the address by reassembling the received fragments. Their basic DPM method has limitations to deal with multiple attackers at the same time with the same source IP address (see Section 4.1). To counter this problem, they propose to produce hash values of the ingress interface and send this hash value along with marked bits. 


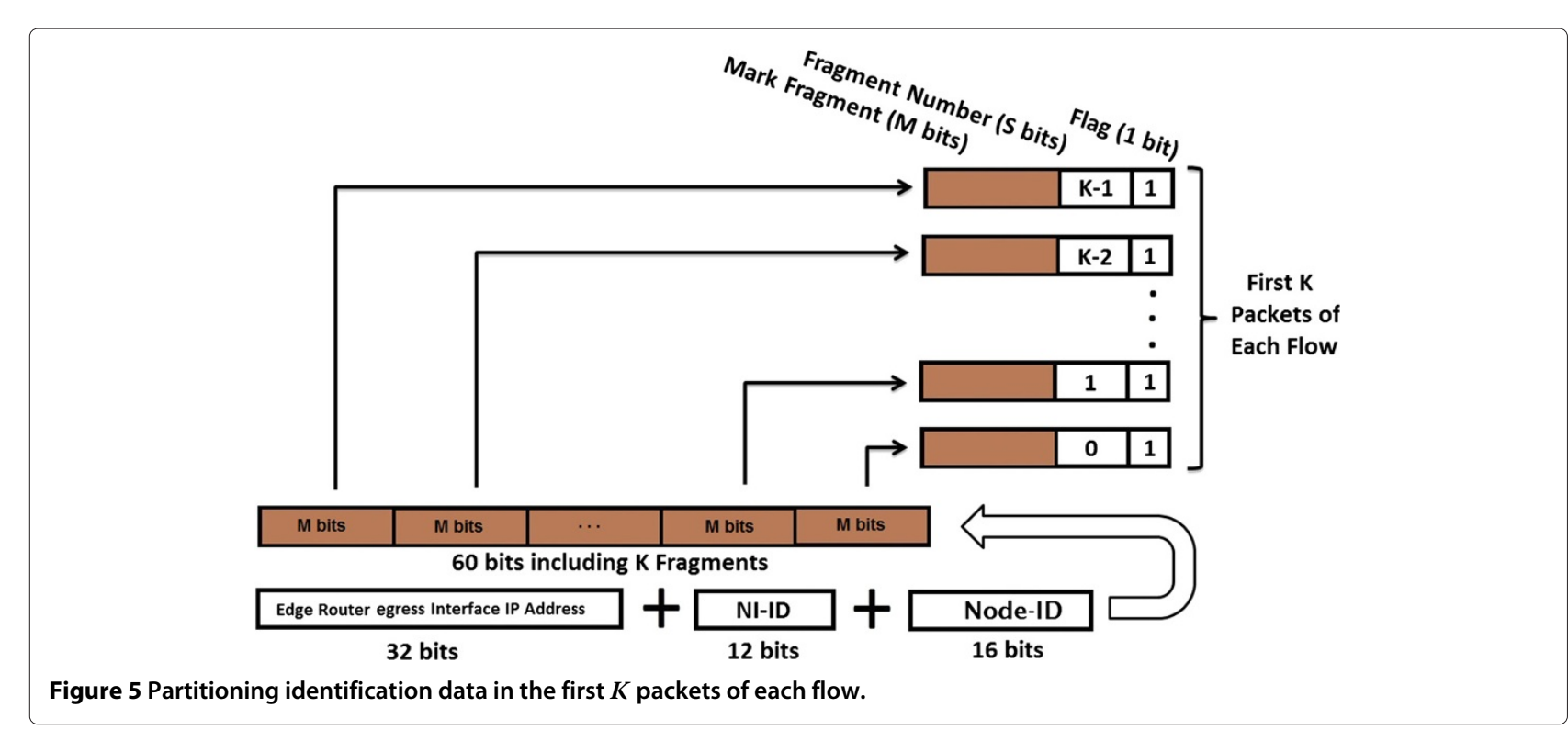

Under the same conditions, our proposed method DFM does not need to use hash functions because DFM first detects the flow to construct a valid mark (see Section 5.3). Thus, DFM is safer to counter the problem of multiple attackers with the same IP addresses at the same time.

Furthermore, in our proposed system, it is assumed that the attacker can change its MAC address. To this end, we have four potential scenarios when an attacker changes his MAC address:

1. The attacker spoofs his MAC address with a random MAC address: In this case, we assumed that MAC filtering is enabled in the edge router, the attacker cannot access to the network.

2. The attacker has access to the white list of MAC addresses and he spoofs his MAC address with an active MAC address: The current switches and routers reject concurrent access of more than one node with the same MAC address, so the attacker cannot access the network.

3. The attacker has access to the white list of MAC addresses and he spoofs his MAC with an inactive MAC address: In this situation, after the attack is detected by the victim, it can block the attacker node, using DFM to distinguish the attacker traffic from the rest of traffic, while the other nodes will still have access to the destination (victim).

4. The attacker spoofs his MAC address with several existing MAC addresses in the white list regularly: After detecting the attack using DFM, the victim assumes that several source nodes from the same network belonging to one interface of the edge router try to send malicious traffic. At this point, the victim traces up to level 2 of traceback (edge router interface) and only cuts off the access of all nodes belonging to this interface, not all nodes that are connected to this edge router. It should be noted here that the other traceback methods in the face of such a situation are only able to trace up to the edge router and therefore, they would cut off the access of all nodes forwarded by the edge router.

As discussed in the first three MAC address changing scenarios, DFM is able to trace three levels up to the attacker node. Only in scenario 4 that the DFM traces two levels up to the source network interface of the edge router. However, this is still much better than the current traceback methods, where they at the best can detect up to the source edge router.

It should be noted here that DFM is able to trace back to the source of the traffic one step behind the ingress interface of the edge router. Every router with a valid IP address on its egress interface can potentially act as an edge router. So if a valid IP address is assigned to the egress interface of the closest router to the local network, then DFM would be able to trace back up to the source node. However, if the network administrator defines the farthest connected router to the Internet as the edge router, then probably, there are some subnets behind that edge router. In this case, DFM is able to trace back up to the sub-networks and therefore, fewer number of routers are required to participate in the DFM marking scheme. This is a trade-off between the accuracy and the number of participant edge routers in the DFM marking scheme.

\subsection{Practical comparison of DFM and DPM}

As described earlier, based on the various IP traceback approaches described in Section 2, DFM and DPM 


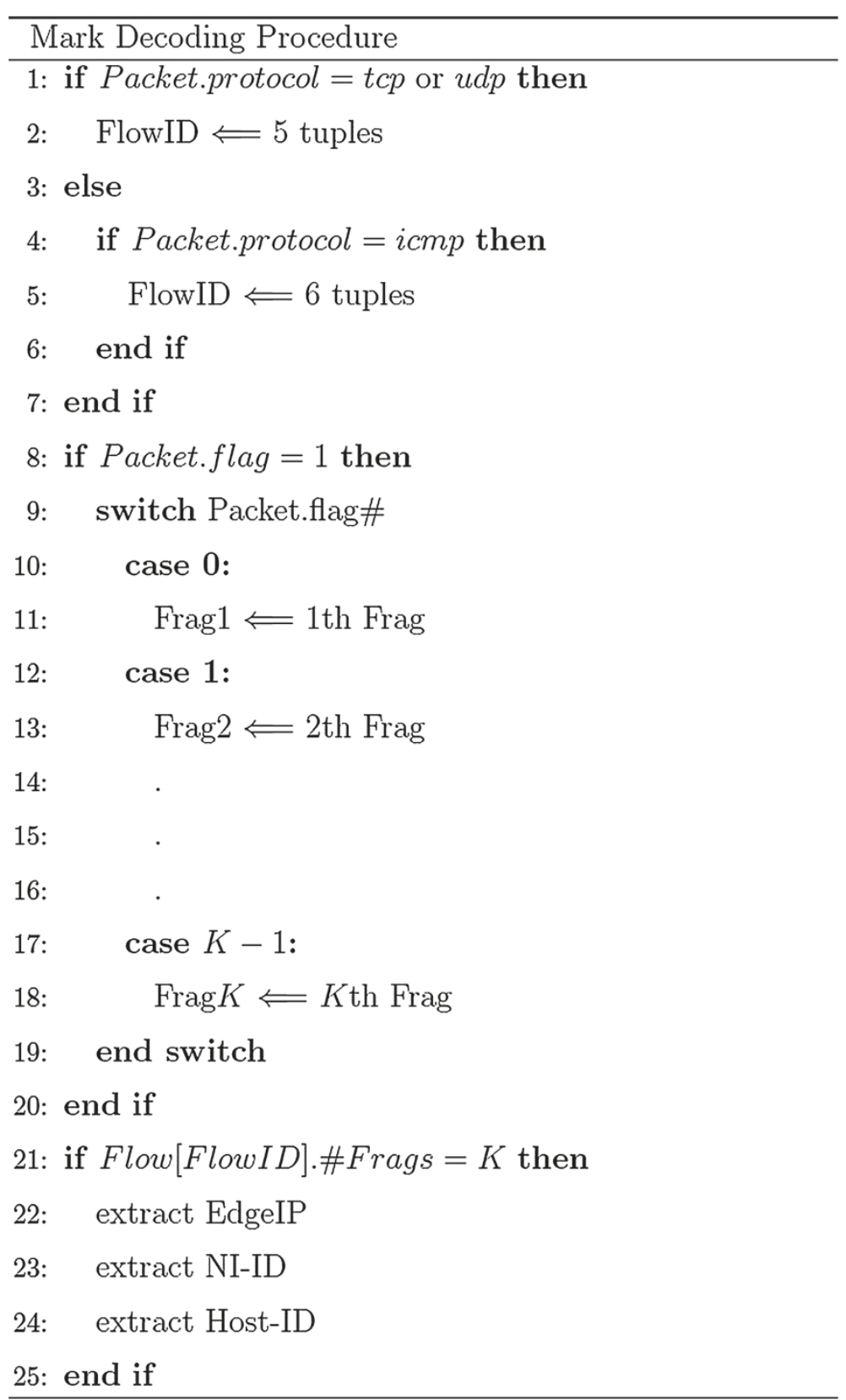

Figure 6 Mark decoding procedure.

fall into the same categories of classification, but PPM falls in the other categories. In addition, the goal of the PPM is entirely different from DPM and DFM. The purpose of PPM is to identify the attack path, while the goal of DPM and DFM is to identify the attack source. So we cannot compare the performance of the PPM directly with the DPM and DFM under the same conditions on the same network. Thus, we only compared the performance of DFM and DPM under the same conditions and on the same network platform [27].
Figure 7 is a schematic illustration of both DPM and DFM approaches, and is a comparison between two methods. To evaluate DFM and compare the result with DPM, we have employed both approaches on five discrete network traces including Darpa 1999 attack and attack-free traces [33], CAIDA anonymized Internet traces October 2012 [34], MAWI traffic archive December 2012 [35], and Dal2010 data sets:

1. Darpa 1999 network traces consists of 5 weeks of traces generated at the MIT Lincoln Lab [33]. The 
Table 4 An example of the reconstruction table with $K=2, M=30$ and $S=1$

\begin{tabular}{|c|c|c|c|}
\hline Flow ID & $\begin{array}{l}\text { First } \\
\text { fragment }\end{array}$ & $\begin{array}{l}\text { Second } \\
\text { fragment }\end{array}$ & Identification \\
\hline \multicolumn{4}{|c|}{ AC100C14055A5585B02A005006 } \\
\hline Srcip = 172.16.112.20 & & & Edge router $\mathrm{IP}=172.16 .0 .1$ \\
\hline SrcPort $=1370$ & AC100001 & $4004005 F$ & $\mathrm{NI}-\mathrm{ID}=1$ \\
\hline Dstip $=85.133 .176 .42$ & & & Host-ID = 23 \\
\hline \multicolumn{4}{|l|}{ DstPort $=80$} \\
\hline \multicolumn{4}{|l|}{ Protocol $=\mathrm{tcp}$} \\
\hline \multicolumn{4}{|c|}{ AC10715410925585B817001511 } \\
\hline Srcip = 172.16.113.84 & & & Edge router $\mathrm{IP}=172.16 .0 .1$ \\
\hline SrcPort $=4242$ & AC100001 & 40080037 & $\mathrm{NI}-\mathrm{ID}=2$ \\
\hline Dstip $=85.133 .184 .23$ & & & Host-ID = 13 \\
\hline \multicolumn{4}{|l|}{ DstPort $=21$} \\
\hline Protocol $=u d p$ & & & \\
\hline
\end{tabular}

first and third weeks do not contain any attacks. The second week contains a selected subset of attacks from the 1998 evaluation in addition to several new attacks. There are 201 instances of about 56 types of attacks distributed throughout forth and fifth weeks data.

2. CAIDA network traces contain anonymized passive traffic traces from CAIDA's Equinix-Sanjose monitor on high-speed Internet backbone links [34]. The

CAIDA data set we employed in this work is a standard tcpdump file from October 2012.

3. MAWI network traces are from a traffic data repository maintained by the MAWI Working Group of the WIDE Project [35]. These traffic traces are in tcpdump format, and the IP addresses in the traces are scrambled because of privacy reasons.

4. Dal2010 network traces were captured in 2010 on the Dalhousie network. Traces were captured from the main Internet link. Given the privacy-related issues, data is filtered to scramble the IP addresses and each packet is further truncated to the end of the IP header so that all payloads are excluded.

Moreover, the checksums are set to zero since they could conceivably leak information from short packets. However, any information regarding size of the packet is left intact.

Table 5 represents statistical information of all of aforementioned network traffic traces.

We implemented a testbed network in our research lab (Figure 8). As shown in this figure, one local network for evaluating our DFM technique on all of the five data sets is given above. For this purpose, we replayed these data sets on our testbed network using tcpreplay and tcprewrite open source applications [36]. In addition, we implemented two real-time programs using Winpcap library by $\mathrm{C}++$ [37], one for marking and the other for tracing back the source of the flows for DFM. The marking program runs at the source edge router and only marks those flows traveling from the inside of the network to the outside. At the same time, the traceback program runs at the destination nodes and tries to trace the source nodes of the marked traffic.

\subsubsection{Traceback and marking rates}

As described before, our marking method divides a 60bit identification data for each flow to $K$ fragments and passes it to the destination by the first $K$ packets of each flow. Therefore, the mark inserted in each packet contains $M=60 / K$ bits of the identification data, $S=\log _{2} K$ bits to identify a fragment and 1 bit flag to identify the marked and the unmarked packets in a flow. However, finding the best number of $K$ is an important issue. There are three metrics that are important in choosing the best value for $K$ :

- TR, traceable rate: the ratio of the number of successfully traced back packets to all packets

- $M R$, marking rate: the ratio of the marked packets by the edge router to all packets

- $N B$, number of bits: the total number of bits that are embedded to the IP header of each mark-carrying packet

Our desirable $K$ should result in higher values of traceback rate (TR) and lower values of marking rate (MR) and number of bits (NB). Table 6 presents the relation 


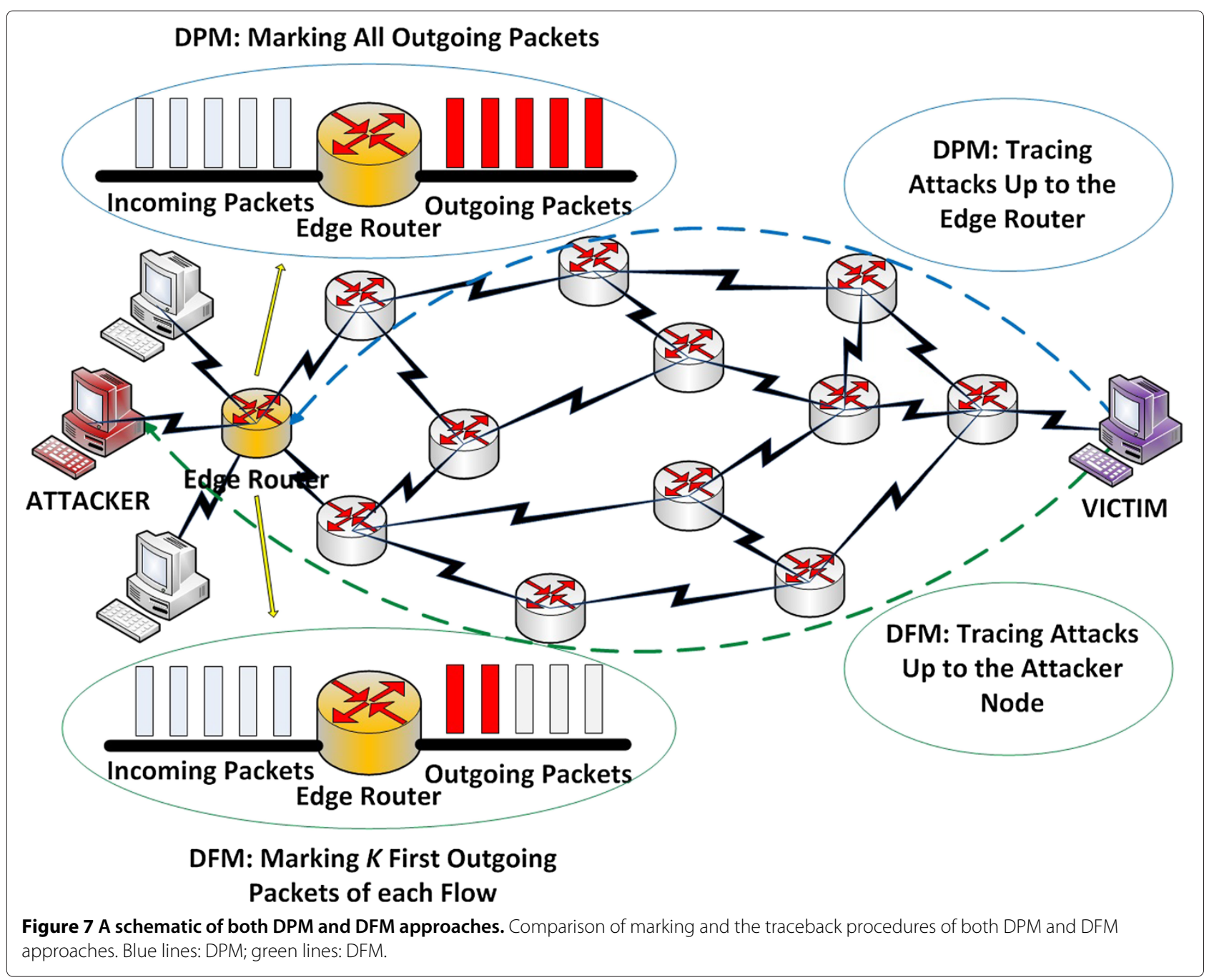

between $K, M, S, \mathrm{NB}$, TR, and MR for all four data sets on DFM approach. For the better understanding of the topic, Figure 9 presents five charts, each chart for one data set showing the values of TR, MR, and normalized NB from 0 to $100 \%$ for several values of $K$ for the proposed DFM approach.

The most interesting thing that can be observed in Figure 9 is that unlike the existing traceback methods where reducing MR causes reducing TR, in DFM reducing
MR increases TR. It means that DFM can achieve a high TR with marking a lower number of packets, i.e., low MR. The reason is that clearly the flows with lower number of $K$ packets are unable to carry all $K$-marked fragments. In this case, decreasing $K$ also decreases MR. As a result, this increases the number of flows that can carry all $K$ fragments and it increases the traceback rate, TR.

However, while decreasing $K$ causes higher TR and lower MR, which are both desirable; it also increases NB,

Table 5 Statistical information of Darpa 1999, CAIDA October 2012, MAWI December 2012 and Dal2010 data sets

\begin{tabular}{lccr}
\hline Data set & Number of packets & Number of flows & Size (byte) \\
\hline Darpa 1999 attack data set & $6,830,176$ & 414,791 & $898,704,130$ \\
Darpa 1999 attack-free data set & $2,765,596$ & 214,114 & $353,421,516$ \\
CAIDA october 2012 & $241,589,706$ & $20,596,858$ & $72,556,397,639$ \\
MAWI 2012 data set & $11,6737,412$ & $6,635,840$ & $3,667,8015,695$ \\
Dal2010 data set & $372,239,644$ & $40,376,143$ & $108,789,054,323$ \\
\hline
\end{tabular}




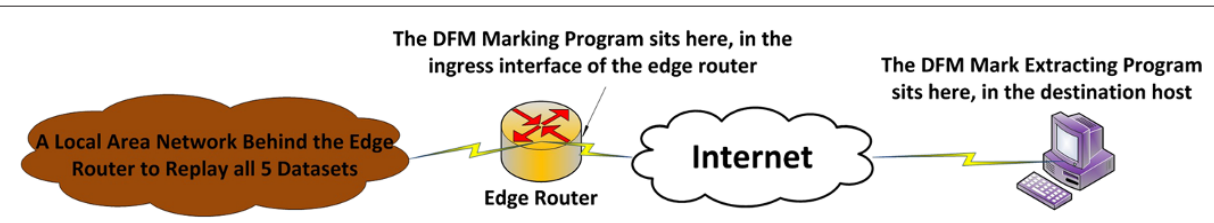

Figure 8 The testbed network to analyze DFM.

which is undesirable. Since TR is a very important factor in DFM, we focus on the values of $K \leq 5$ that reasonable traceback rates (around $90 \%$ and more). On the other hand, $K=1$ cannot be an option because in that case the required NB becomes 61 bits. It should be noted here that finding 61 rarely usable bits in an IP header to embed marking bits is almost impossible. Selecting $K$ values from 2 to 5 is a trade-off between MR and NB. While lower $K$ values have better MR and worse NB, higher $K$ values have worse MR and better NB. As most traceback methods utilize 16 bits of identification field in the IP header to embed the marking bits $[11,13,15-17], K=5$ seems to be a good option. It requires 16 bits of IP header and around $12 \%$ to $33 \%$ of all packets end up being marked. However, in our implementation it is possible to set $K=2$. In this case, we have better MR compared to $K=5$ (around $8 \%$ to $15 \%$ ), but more NB (32 bits). Based on the previous works on IP traceback $[9,21]$, it is possible to use the identification, the flag and the fragment offset fields of IP header as a 32-bit marking field (Figure 10). Fortunately, the use of the fragment and the identification fields in the IP header affects only the $0.06 \%$ of the legitimate packets $[9,21]$.
It should be noted here that each flow requires at least $K$ packets to carry all the marking fragmentations. So if the number of packets in a flow is less than $K$, then this flow cannot be traced back by DFM. This is the reason why changing the $K$ changes the TR in Table 6 .

Our results show that marking the first two packets of every outgoing flow using DFM makes it possible to correctly determine the origin of $93 \%$ to $99 \%$ of packets (TR), while it only requires $9 \%$ to $15 \%$ of all packets to be marked (MR). Moreover, DFM correctly determines the origin of $90 \%$ to $99 \%$ of packets (TR) by marking $12 \%$ to $33 \%$ of all packets (MR) if the first five packets of every outgoing flow are marked (italic entries in Table 6).

Table 7 shows the evaluation of the DPM approach on all of our five evaluation data sets, using the same TR and MR metrics as we used to evaluate the DFM approach. Although it is expected to have $100 \%$ traceback rate using DPM approach, actually as it is shown in Table 7, TR for the DPM approach is less than 100\% because fragmented traffic will be corrupted by the DPM and there is some fragmented traffic in our evaluation data sets. If a single fragment of the original datagram

Table 6 The relationship between $K, M, S, N B, T R$, and MR on all evaluation data sets for the DFM approach

\begin{tabular}{|c|c|c|c|c|c|c|c|c|c|c|c|c|c|}
\hline \multirow[b]{2}{*}{$K$} & \multirow[b]{2}{*}{$M$} & \multirow[b]{2}{*}{$S$} & \multirow[b]{2}{*}{$\begin{array}{c}\text { NB } \\
(M+S+1)\end{array}$} & \multicolumn{2}{|c|}{$\begin{array}{c}\text { Darpa } 1999 \\
\text { attack }\end{array}$} & \multicolumn{2}{|c|}{$\begin{array}{l}\text { Darpa } 1999 \\
\text { attack-free }\end{array}$} & \multicolumn{2}{|c|}{$\begin{array}{c}\text { CISDA } \\
\text { Oct } 2012\end{array}$} & \multicolumn{2}{|c|}{$\begin{array}{c}\text { MAWI } \\
\text { Dec } 2012\end{array}$} & \multicolumn{2}{|c|}{ Dal2010 } \\
\hline & & & & TR & MR & $\mathrm{TR}$ & MR & TR & MR & TR & MR & $\mathrm{TR}$ & MR \\
\hline 1 & 60 & 0 & 61 & 100 & 7.74 & 100 & 6.07 & 100 & 8.52 & 100 & 5.68 & 100 & 10.85 \\
\hline 2 & 30 & 1 & 32 & 98.65 & 14.13 & 99.94 & 12.09 & 92.91 & 9.96 & 97.23 & 8.60 & 93.33 & 15.02 \\
\hline 3 & 20 & 2 & 23 & 98.62 & 20.51 & 99.94 & 18.11 & 91.76 & 10.82 & 96.96 & 11.38 & 92.53 & 18.79 \\
\hline 4 & 15 & 2 & 18 & 98.61 & 26.89 & 99.88 & 24.11 & 91.03 & 11.43 & 96.84 & 14.12 & 91.46 & 22.21 \\
\hline 5 & 12 & 3 & 16 & 98.24 & 33.18 & 99.45 & 30.00 & 90.56 & 11.93 & 96.20 & 16.70 & 89.77 & 25.21 \\
\hline 6 & 10 & 3 & 14 & 78.64 & 35.54 & 81.73 & 32.35 & 90.11 & 12.34 & 92.88 & 18.62 & 87.11 & 27.67 \\
\hline 10 & 6 & 4 & 11 & 66.98 & 39.66 & 70.97 & 36.77 & 88.82 & 13.40 & 86.06 & 23.17 & 76.98 & 33.36 \\
\hline 12 & 5 & 4 & 10 & 64.55 & 40.63 & 68.65 & 37.98 & 88.48 & 13.78 & 84.27 & 24.75 & 74.63 & 35.07 \\
\hline 15 & 4 & 4 & 9 & 60.83 & 41.26 & 64.99 & 38.98 & 88.08 & 14.28 & 81.74 & 26.55 & 72.13 & 37.07 \\
\hline 20 & 3 & 5 & 9 & 59.71 & 41.69 & 63.38 & 39.94 & 87.64 & 14.98 & 77.84 & 28.37 & 68.35 & 39.40 \\
\hline 30 & 2 & 5 & 8 & 59.27 & 42.28 & 62.21 & 41.32 & 87.12 & 16.15 & 74.73 & 30.66 & 64.82 & 42.35 \\
\hline 60 & 1 & 6 & 8 & 58.49 & 43.43 & 58.90 & 43.51 & 85.95 & 18.91 & 70.76 & 34.14 & 59.50 & 46.98 \\
\hline
\end{tabular}

DFM correctly determines the origin of $90 \%$ to $99 \%$ of packets (TR) by marking $12 \%$ to $33 \%$ of all packets (MR) if the first five packets of every outgoing flow are marked (italic entries). 


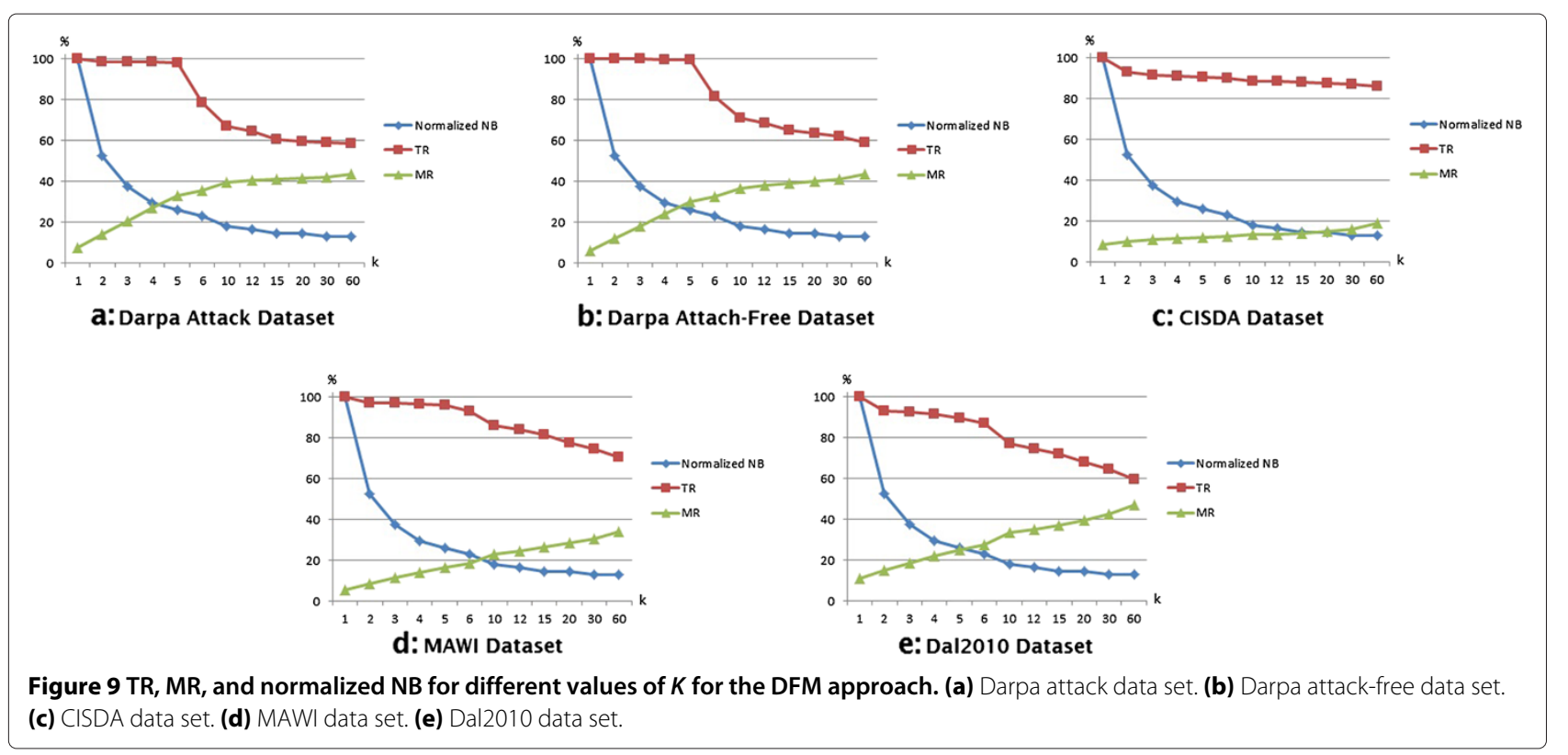

is marked, the reassembly function would fail at the destination.

By comparing Tables 6 and 7, it can be seen that DPM has higher traceback rate compared to DFM (TR); however, this accuracy is achieved by marking all the packets in the network (MR).

\subsubsection{Memory usage}

The following are the memory usage comparison between the DFM and the DPM approaches:

- Memory usage of DFM approach in the edge router. The space required for running DFM on an edge router is equal to the sum of the required space of three tables namely the flow table, the NI-ID table, and the Node-ID table [26]. In our practical (on our testbed) analysis, the total required space for running the DFM method was less than $26 \mathrm{~KB}$. Below we explain the details of each of these tables and the space required by them:

\begin{tabular}{|c|c|c|c|c|c|}
\hline Bit offset & $0-3$ & $4-7$ & $8-15$ & $16-18$ & $19-31$ \\
\hline 0 & Version & Header Length & TOS & \multicolumn{3}{|c|}{ Total Length } \\
\hline 32 & \multicolumn{3}{|c|}{ Identification field } & Flag & Fragment offset \\
\hline 64 & \multicolumn{3}{|c|}{ TL } & Protocol & \multicolumn{2}{|c|}{ Header checksum } \\
\hline 96 & \multicolumn{4}{|c|}{ Source address } \\
\hline 128 & \multicolumn{3}{|c|}{ Destination address } \\
\hline 168 & \multicolumn{3}{|c}{ Options } \\
\hline 160 & \multicolumn{3}{|c}{ Payload (First 8 bytes) } \\
$196+$ & \multicolumn{3}{|c|}{} \\
\hline
\end{tabular}

Figure 10 Using the gray fields as marking field in IP header for $K=2$.
- NI-ID table. For every interface on the edge router and for every VLAN (in case of existence of VLANs on the edge router), 9 bytes including 12 bits for NI-ID, 48 bits for MAC address, and 12 bits for VLAN ID are stored. Since the implemented evaluation network has assigned one interface for evaluating the DFM approach (Figure 8), this table only requires 9 bytes in the edge router.

- Node-ID table. For every record in the NI-ID table, DFM stores a separate Node-ID table. For every new observed source MAC address, a 60-bit record including 12 bits NI-ID and 48 bits MAC address should be stored. Thus, the size of this table varies and is based on the number of unique source MAC addresses that are observed. The DFM approach utilizes a memory management algorithm, so when it does not observe a source MAC address for a specific period of time, it removes its record from the Node-ID table. In our experimental results, the maximum required space for storing the Node-ID table was 945 bytes.

- Flow table. In addition to NI-ID and Node-ID tables, DFM utilizes another table called the

Table 7 TR and MR of DPM approach for all five evaluation data sets

\begin{tabular}{cccccc}
\hline $\begin{array}{c}\text { Data } \\
\text { set }\end{array}$ & $\begin{array}{c}\text { Darpa 1999 } \\
\text { attack }\end{array}$ & $\begin{array}{c}\text { Darpa 1999 } \\
\text { attack-free }\end{array}$ & $\begin{array}{c}\text { CISDA } \\
\text { Oct 2012 }\end{array}$ & $\begin{array}{c}\text { MAWI } \\
\text { Dec 2012 }\end{array}$ & Dal2010 \\
\hline TR & 99.83 & 99.98 & 99.26 & 99.42 & 99.33 \\
MR & 100 & 100 & 100 & 100 & 100 \\
\hline
\end{tabular}


flow table. Each row in this table belongs to an observed flow. DFM stores 180 bits for each flow including the following three items:

* Flow ID, 13 bytes. For TCP and UDP flows, the flow ID is the sum of five-tuples including 4 bytes source IP addresses, 2 bytes source port numbers, 4 bytes destination IP addresses, 2 bytes destination port numbers, and 1 byte of protocol, which makes 13 bytes in total. For ICMP flows, the flow ID is the sum of six-tuples including 4 bytes source IP addresses, 4 bytes destination IP addresses, 1 byte protocol, 1 byte ICMP type, 1 byte ICMP code, and 2 bytes ICMP ID, altogether 13 bytes.

* Flow mark, 60 bits as described earlier.

* Packet number, 2 bytes. The edge router increases this number by one in the corresponding flow record for every transmitted packet. In other words, this number indicates the number of packets in a flow. DFM uses this number for keeping track of $K$ first packets of every flow.

DFM no longer needs keeping a record of a flow when the flow is over. End of a flow is detected by an interpacket delay that is more than $600 \mathrm{~ms}$. Therefore, the space required for flow table varies and is based on the number of concurrent flows. Table 8 shows the size of the flow table in the DFM approach for each of the five evaluation data sets. Since the maximum number of concurrent flows was 1131, the maximum required space to store the flow table was about $25 \mathrm{~KB}$ on our testbed.

- Memory usage of DFM approach at the victim side. The victim maintains a reconstruction table, matching the flow ID and $K$ possible mark fragments. For every observed flow, a 13 bytes flow ID and 60 bits identification data should be stored. Like the flow table in the edge router, the victim no longer needs keeping the record of a flow when a flow is over. Therefore, the space required for the reconstruction table varies and is based on the number of concurrent flows. Table 8 shows the size of the reconstruction table in DFM approach for each of the five evaluation data sets. Since the maximum number of concurrent flows was 1,131, the maximum required space to store the reconstruction table was about $23 \mathrm{~KB}$.
- Memory usage of DPM approach in the edge router. Since marking process on the edge router by the DPM approach only stores the hash value of the ingress IP address, router's memory overhead in DPM algorithms is negligible.

- Memory usage of DPM approach at the victim side. The reconstruction table consists of $f$ parts, and each of those parts is a 217-bit structure $\left(2^{d}\right.$ areas, $k$ segments in every area, and $2^{a}$ bits in every segment) [20]. $f$ Refers to the number of hash value functions. We implemented the DPM approach with the suggested four hash value functions [20]. Therefore, the required space for reconstruction table was $64 \mathrm{~Kb}$.

\subsection{Authenticated flow marking}

Using DFM, the destination is able to trace up to the source node of the received traffic by extracting the edge router's IP Address, NI-ID, and Node-ID from the marked packets of each flow. Although DFM has promising results, in case of a compromised router on a network path, the marking bits could be changed and the destination would be unable to identify the origin of the traffic.

To this end, we propose to add an optional digital signing mechanism to DFM. A straightforward approach for the digital signing is to use the RSA algorithm. However, RSA has two major disadvantages. Firstly, it is very expensive to compute. Secondly, the memory overhead is large (128 bytes for a 1,024-bit RSA signature). Thus, we choose to use the Elliptic Curve Digital Signature Algorithm (ECDSA) [38]. Elliptic curve systems offer more security per bit increase in the key size compared to RSA or Diffie-Hellman public key systems. A 160-bit elliptical curve key is equivalent to a 1,024-bit RSA key in terms of security. Security is not the only attractive feature of elliptic curve cryptography. Elliptic curve cryptosystems also are more computationally efficient than the RSA and Diffie-Hellman systems [39].

If we assume that each edge router shares its own ECDSA public key, e.g., through a certificate authority (CA), each edge router is able to create a signature for every flow that travels from the inside (of the network) to the outside with its own private key. Then it can send signatures along with the marking data to the destinations. Because a compromised router does not know the secret keys of the edge routers, it cannot forge flow markings. The flow-specific information is necessary to prevent a replay attack because otherwise, a compromised router can forge other routers markings simply by copying their mark and signing into other flows. As flow-specific information, we could use the flow ID. 
Table 8 The size of the flow table in the DFM approach for each of the five evaluation data sets

\begin{tabular}{lccccc}
\hline Data set & $\begin{array}{c}\text { Darpa 1999 } \\
\text { attack }\end{array}$ & $\begin{array}{c}\text { Darpa 1999 } \\
\text { attack-free }\end{array}$ & $\begin{array}{c}\text { CISDA } \\
\text { Oct 2012 }\end{array}$ & $\begin{array}{c}\text { MAWI } \\
\text { Dec 2012 }\end{array}$ & $\begin{array}{c}\text { Dal2010 } \\
\text { Number of concurrent flows }\end{array}$ \\
Flow table size at the edge router (byte) & 18.5 & 241 & 1,131 & 930 & 1,021 \\
Reconstruction table size at the destination (byte) & 17 & 5.5 & 25 & 20.5 & 22.5 \\
\hline
\end{tabular}

The required space for each flow in the flow table is 180 bits and in the reconstruction table is 164 bits (13 bytes flow ID and 60 bits identification data).

To this end, the edge router creates 42 bytes signature value (size of ECDSA digital signature with 160 bits elliptical curve key) by applying ECDSA signing algorithm to 60 bits identification data plus 13 bytes flow ID. This 42 bytes signature should also transfer to the destination along with 60 bits of identification data. Obviously, it is not a good idea to embed 42 bytes of data to the IP header because embedding 42 bytes data and 60 bits identification data to 32 bits marking field requires at least 13 packets, while looking at Table 6 shows that $K=13$ has a bad TR (64\% to $88 \%$ ). So we decided to add 42 bytes ECDSA digital signature to the end of the $K$ th packet payload of each flow. It is assumed that when this system is used it would be known that DFM is using ECDSA signing algorithm.

\subsection{Signature verification by destination}

As described earlier, each destination maintains a reconstruction table (Table 4). In the case of authenticated flow marking, each destination should add two more fields to this table: one field for ECDSA digital signature data and another field for the signature verification status (Table 9). We assume that the destination already has the public key of each edge router that is participated in the authenticated flow marking method, e.g., by downloading the public key from the CA. When the destination gets the signed flow, it uses the sender's public key to authenticate the sender. If the two agree, the destination knows that the author of the mark was in possession of the edge router's private key and that the mark is in fact valid; otherwise, it would reject the flow.

One advantage of the proposed authenticated flow marking method is that it is optional for the destination to extract and validate the signature for every flow while it does not get attacking flows. In a situation when the victim is under attack, it may use the signature to validate the mark to find the attacker node. Therefore, the destination is not forced to always consume its CPU and memory resources to verify ECDSA signature.

5.8 Experimental results of authenticated DFM evaluation To evaluate our proposed authentication DFM method, we applied this method on the same five data sets as used above. To this end, we modified our original DFM implementation in both the attacker and the victim sides to include the authentication component. In this case, the program at the source (attacker) side has optional ECDSA digital signature embedding with 160-bit elliptical curve key as well as the flow marking component. Whereas the program at the destination side has optional edge router

Table 9 An example of the reconstruction table for the DFM authenticated flow marking method

\begin{tabular}{|c|c|c|c|c|c|}
\hline Flow ID & First mark frag & Second mark frag & Digital sign & Identification & Sign verification \\
\hline \multicolumn{6}{|l|}{ AC100C14055A5585 } \\
\hline \multicolumn{6}{|l|}{ B02A005006 } \\
\hline Srcip $=172.16 .112 .20$ & & & & EdgeRouter IP = & \\
\hline SrcPort $=1370$ & AC100001 & $4004005 F$ & 42 bytes & 172.16.0.1 & Verified \\
\hline Dstip $=85.133 .176 .42$ & & & sign data & $\mathrm{NI}-\mathrm{ID}=1$ & \\
\hline DstPort $=80$ & & & & Host-ID $=23$ & \\
\hline \multicolumn{6}{|l|}{ Protocol $=\mathrm{tcp}$} \\
\hline \multicolumn{6}{|l|}{ AC10715410925585 } \\
\hline \multicolumn{6}{|l|}{ B817001511 } \\
\hline Srcip $=172.16 .113 .84$ & & & & EdgeRouter IP = & \\
\hline SrcPort $=4242$ & AC100001 & 40080037 & 42 bytes & 172.16.0.1 & Failed \\
\hline Dstip $=85.133 .184 .23$ & & & sign data & $\mathrm{NI}-\mathrm{ID}=2$ & \\
\hline DstPort $=21$ & & & & Host-ID = 13 & \\
\hline Protocol $=u d p$ & & & & & \\
\hline
\end{tabular}


verification as well as extracting marks, using crypto++ library in $\mathrm{C}++[40]$. The program at the attacker side runs at the edge router and only marks and signs those flows traveling from the inside of the network to the outside. At the same time, the traceback program runs at the destination node and tries to detect the source of the marked traffic and verifies the edge router.

\subsubsection{Bandwidth usage}

It is clear that enabling the optional edge router authentication increases the network bandwidth usage given that an extra 42 bytes signature data is embedded to the end of the $K$ th packet of each flow. The amount of this increase can be observed by comparing the size of the transmitted traffic with and without the flow signing (Table 10). This comparison shows that performing the optional edge router authentication and the verification has less than $2 \%$ bandwidth overhead.

\subsubsection{Computational cost}

To investigate the processing overhead of the edge router that has direct impact on the network transmission delay, we estimated the ability of our proposed method by computing the signing and verification on a 164-bit message consisting of 60 bits identification data and the 13 bytes flow ID. This estimation is based on running our algorithm on a $\mathrm{PC}$ with $3.4 \mathrm{GHz}$ processor and Ubuntu 10.04 operating system. Our experimental results show that signing a flow at the source edge router takes about $0.38 \mathrm{~ms}$ and verifying the digital signature at the destination takes about $0.9 \mathrm{~ms}$. Thus, our authenticated flow marking algorithm takes less than $1.3 \mathrm{~ms}$.

\subsubsection{Memory usage}

Since the digital signature of a flow is created and embedded to the flow at the time of sending the flow, no signature data is stored at the edge router. Thus, performing the authenticated flow marking method does not need extra memory.

In summary, it is clear from these experimental results that the DFM has the ability of tracing traffic to its origin behind an edge router with high accuracy in practice. This is achieved only by marking approximately $9 \%(K=2$ in MAWI data set) to $33 \%$ ( $K=5$ in Darpa attack data set) of all transmitted packets. In addition, our authenticated flow marking method guaranties that the flow marking is not changed in the network transmission path by a forged router.

\subsection{DFM analysis}

To analyze the DFM, we have used the same evaluation metrics that we used to analyze the PPM and the DPM in the following sections.

\subsubsection{Computational overhead}

Like DPM, in DFM only the closest edge router to the attacker is responsible for marking, and there are some computational overhead such as preparing marking information and upgrading marking fields. However, unlike DPM, DFM does not require to calculate the hash value of the ingress address. Moreover, unlike DPM that extracts hash values of ingress addresses, the victim uses the flow ID as a guide to prevent the combinatorial explosion problem [20] in DPM. In addition, unlike DPM, DFM only marks $K$ first packets of each flow, not all packets, and the victim extracts marking information from only those packets that the flag bit is set, again not all packets. Therefore, DFM has lower computational overhead than DPM. Moreover, as previously discussed, signing a flow takes less than half a millisecond and verifying the digital sign of a flow takes less than one millisecond by the authenticated DFM algorithm.

Table 10 The size of the transmitted traffic with and without the source edge router authentication in bytes

\begin{tabular}{llccc}
\hline Data set & K & Marking without authentication & Marking with authentication & Increment (\%) \\
\hline Darpa attack & 2 & $353,421,516$ & $360,828,636$ & 2.09 \\
& 5 & $353,421,516$ & $359,394,340$ & 1.69 \\
Darpa attack-free & 2 & $898,704,130$ & $915,913,630$ & 1.91 \\
& 5 & $898,704,130$ & $912,723,914$ & 1.56 \\
CISDA & 2 & $72,556,397,639$ & $72,701,839,187$ & 0.2 \\
& 5 & $72,556,397,639$ & $72,618,776,795$ & 0.08 \\
MAWI & 2 & $3,667,8015,695$ & $36,821,053,121$ & 0.39 \\
& 5 & $3,667,8015,695$ & $36,804,518,141$ & 0.34 \\
Dal2010 & 2 & $108,789,054,323$ & $109,441,443,893$ & 0.60 \\
& 5 & $108,789,054,323$ & $109,257,225,173$ & 0.43 \\
\hline
\end{tabular}




\subsubsection{Memory overhead}

Like PPM and DPM, memory overhead on the routers in DFM is negligible (about $25 \mathrm{~Kb}$ ), and at the victim side, DFM requires a small reconstruction table $(23 \mathrm{~Kb})$. This is even less than the DPM requirements $(64 \mathrm{~Kb})$.

\subsubsection{False positives}

As discussed earlier, the DPM algorithm uses a limited number of bits for storing a hash value to prevent the combinatorial explosion problem. However, this then results in false positives in tracebacking an IP address under DDoS attacks when the number of attacks are more than the DPM can handle. On the other hand, the DFM algorithm uses the flow ID to prevent the combinatorial explosion problem. Therefore, it does not face the false-positive rates problem under the DDoS attacks.

\subsubsection{Mark spoofing by attackers}

DFM marks each flow when it enters to the edge router. In this case, even if an attacker tries to spoof the mark, the spoofed mark will be overwritten with a correct mark, once the flow passes through the edge router. Therefore, mark spoofing by the attacker is not an issue for the DFM approach.

\subsubsection{Mark spoofing by subverted routers}

Unlike PPM and DPM, which do not have any solution to counter the malfunctioned routers in the attack path, DFM has an optional authenticated flow making feature to ensure that the marking information have not been changed in the network path.

\subsubsection{Awareness of the attack path length in advance} Unlike PPM, the process of trackback in DFM approach does not need the path length; therefore, awareness of the attack path length is not an issue.

\subsubsection{Awareness of the network map and the routing in advance}

Unlike PPM, the goal of DFM is not to reconstruct the attack path, so awareness of the network map and the routing is not an issue for DFM.

\subsubsection{The number of required packets for traceback}

With the suggested $N B=32$, the DFM requires two packets and with $N B=16$, and DFM requires five packets to trace back up to the attacker node [26]. This is lower than eight packets required by DPM to trace back to the ingress interface address of the edge router [20] and much lower than at least 1,000 packets required by PPM to find the attack path [13].

\subsubsection{Fragmentation}

Like PPM and DPM, the DFM uses the ID field in the IP header of the packets, which is generally used for fragmentation. Thus, if only a single packet of a fragmented datagram is marked, then the datagram reassembly will fail.

\subsubsection{ISP involvement}

Like DPM, the involvement of ISPs is very limited. Only the edge routers have to be upgraded to support the function of deterministic packet marking, and the other routers in the attack path and the network backbone do not need to be responsible for any function of the DPM traceback process.

\subsection{Discussion}

In addition to all the advantages of the DFM that are discussed above, there is one more unique feature that does not exist in any other traceback method. This is to enable the victim to trace the attack source, not only up to the source edge routers, but also to the exact source network interface of the edge router and then, to the source node(s) located in a local areas network behind the edge routers. DFM assumes that each node in a local network may change its IP address, and the MAC filtering is enabled in the edge router. Moreover, the attacker may change its MAC address. However, in these cases, if the attacker changes his MAC address, DFM is still able to trace three levels up to the attacker node. Only in a case when the attacker spoofs his MAC address with several existing MAC addresses in the white list regularly, then the DFM can trace two levels up to the source network interface of the edge router.

Finally, as discussed earlier, using the proposed authenticated flow marking method is optional for the destination in the DFM approach. In a situation when the victim is under attack, it may use the signature to validate the mark to find the attacker node, otherwise the destination is not forced to consume its CPU and memory resources to verify ECDSA signature.

\section{Conclusion}

In this work, we presented a survey of two schemes for IP traceback, the well-known probabilistic packet marking, PPM, and the deterministic packet marking, DPM, approaches. Then we presented the deterministic packet marking, DFM, a novel real-time three-level traceback method. We performed an evaluation and a comparison of all three IP traceback techniques from the perspective of practicality and feasibility. For this evaluation we employed five different network traffic traces including Darpa 1999 attack and attack free, CAIDA Internet traces October 2012, MAWI December 2012, and Dalhousie 2010 data sets, and used several metrics to evaluate the performance of disparate traceback schemes. The metrics employed to evaluate the performance of disparate traceback schemes are the computational overhead, the memory overhead, the bandwidth overhead, the traceback 
Table 11 Comparison of PPM, DPM, and DFM

\begin{tabular}{|c|c|c|c|c|}
\hline Comparison metrics & PPM & DPM & Basic DFM & Authenticated DFM \\
\hline 1. Packet marking rate (MR) & Low & $100 \%$ & $9 \%$ to $33 \%$ & $9 \%$ to $33 \%$ \\
\hline $\begin{array}{l}\text { 2. Mark spoofing by } \\
\text { subverted routers }\end{array}$ & Yes & Yes & Yes & No \\
\hline $\begin{array}{l}\text { 3. Maximum traceback } \\
\text { ability }\end{array}$ & Edge router & Ingress interface & Attacker node & Attacker node \\
\hline 4. Mark spoofing by attacker & Yes & No & No & No \\
\hline $\begin{array}{l}\text { 5. Computational overhead } \\
\text { on routers }\end{array}$ & Low & Low & Low & Fair \\
\hline $\begin{array}{l}\text { 6. Computational overhead } \\
\text { on victim }\end{array}$ & High & Low & Low & Fair \\
\hline $\begin{array}{l}\text { 7. Memory overhead } \\
\text { on routers }\end{array}$ & Low & Low & Low & Low \\
\hline $\begin{array}{l}\text { 8. Memory overhead } \\
\text { on victim }\end{array}$ & High & Low & Low & Low \\
\hline 9. Bandwidth overhead & No & No & No & Low \\
\hline 10. Traceback rate (TR) & Low & Good & Fair & Fair \\
\hline 11. False-positive rate & High & $\begin{array}{l}\text { Low, except heavy } \\
\text { DDoS attacks }\end{array}$ & Low & Low \\
\hline $\begin{array}{l}\text { 12. Number of required } \\
\text { packets for traceback }\end{array}$ & 1,000 & 8 & 2 or 5 & 2 or 5 \\
\hline $\begin{array}{l}\text { 13. Awareness of the attack } \\
\text { path length in advance }\end{array}$ & Yes & No & No & No \\
\hline $\begin{array}{l}\text { 14. Awareness of the } \\
\text { network map and } \\
\text { routing in advance }\end{array}$ & Yes & No & No & No \\
\hline 15. ISP involvement & High & Low & Low & Low \\
\hline $\begin{array}{l}\text { 16. Ability to handle } \\
\text { fragmentation }\end{array}$ & No & No & No & No \\
\hline $\begin{array}{l}\text { 17. Ability to handle major } \\
\text { DDoS attacks }\end{array}$ & Poor & Fair & Good & Good \\
\hline 18. Number of marking bits & 16 & 17 & $\begin{array}{l}\text { If } K=2: 16 \\
\text { If } K=5: 32\end{array}$ & $\begin{array}{l}\text { If } K=2: 16 \\
\text { If } K=5: 32\end{array}$ \\
\hline
\end{tabular}

rate, the false-positive rates, mark spoofing by attackers or subverted routers in the attack path, awareness of the attack path length, network map and routing in advance, the number of required packets for traceback, the percentage of marked packets, Internet service providers (ISP), the ability to handle fragmentation, the ability to handle major DDoS attacks, and the maximum traceback ability. Table 11 provides a summary of the evaluation and offers a comparison of all three IP traceback techniques. The results show that DFM reduces the required number of packets for tracebacking accurately up to $91 \%$ on average with no false positives. Furthermore, the optional authentication scheme for DFM provides efficient authentication of routers' markings such that even a compromised router cannot forge or tamper markings from other uncompromised routers. This optional feature only consumes less than $2 \%$ of the network bandwidth and it takes less than $1.3 \mathrm{~ms}$ delay to one packet of every flow, which is negligible even in streaming traffic such as voice or video [41]. Moreover, DFM traces the attack source up to the attacker node, even if the attack has been originated from a network behind a NAT server. Future work will include a more detailed experimentation of the relationship between the DFM accuracy and the number of 
participating routers. Moreover, we will explore how to embed an IP traceback scheme such as DFM into existing security systems and frameworks.

\section{Competing interests}

The authors declare that they have no competing interests.

\section{Acknowledgements}

This research is supported by the Natural Science and Engineering Research Council of Canada (NSERC) grant and is conducted as part of the Dalhousie NIMS Lab at http://projects.cs.dal.ca/projectx/.

Received: 30 July 2013 Accepted: 10 October 2013

Published: 9 November 2013

\section{References}

1. Z Gao, N Ansari, Tracing cyber attacks from the practical perspective. IEEE Communications Magazine. 43(5), 123-131 (2005)

2. T Subbulakshmi, IAA Guru, SM Shalinie, in International Conference on Recent Trends in Information Technology (ICRTIT), Chennai, 3-5 June 2011. Attack source identification at router level in real time using marking algorithm deployed in programmable routers, (2011), pp. 79-84

3. S Matsuda, T Baba, A Hayakawa, T Nakamura, in Proceedings of the 2002 Symposium on Applications and the Internet. Design and implementation of unauthorized access tracing system, (2002), pp. 74-81

4. AC Snoeren, C Partridge, LA Sanchez, CE Jones, F Tchakountio, B Schwartz, ST Kent, WT Strayer, Single-packet IP traceback. IEEE/ACM Transactions on Networking. 10(6), 721-734 (2002)

5. M Sung, J Li, J Xu, L Li, Large-scale IP traceback in highspeed internet: practical techniques and theoretical foundation. IEEE/ACM Transactions on Networking. 16(6), 1253-1266 (2008)

6. A Belenky, N Ansari, On IP traceback. IEEE Communications Magazine. 41(7), 142-153 (2003)

7. S Bellovinm, IETF Draft on ICMP traceback messages. March 2000. http:// tools.ietf.org/html/draft-bellovin-itrace-00

8. S Savage, D Wetherall, A Karlin, T Anderson, Practical network support for IP traceback. IEEE/ACM Transactions on Networking. 9(3), 226-237 (2001)

9. Z Gao, N Ansari, A practical and robust inter-domain marking scheme for IP traceback. The International Journal of Computer and Telecommunications Networking. 51(3), 732-750 (2007)

10. A Mankin, D Massey, C Wu, SF Wu, Zhang L, On design and evaluation of intention-driven, "Intention-Drive" ICMP Traceback. Tenth International Conference on Computer Communications and Networks, 159-165 (2001)

11. S Savage, A Karlin, D Wetherall, T Anderson, Network support for IP traceback. IEEE/ACM Transactions on Networking, 9(3), 226-237 (2001)

12. D Dean, M Franklin, A Stubblefield, An algebraic approach to IP traceback. ACM Transactions on Information and System Security (TISSEC). 5(2), 119-137 (2002)

13. DX Song, A Perrig, Advanced and authenticated marking schemes for IP traceback. IEEE Computer and Communications INFOCOM. 2, 878-886 (2001)

14. TW Doeppner, P Klein, A Koyfman, in Proceedings of the 7th ACM Conference on Computer and Communications Security CCS. Using router stamping to identify the source of IP packets, (2000), pp. 184-189

15. Y Tseng, H Chen, W Hsieh, Probabilistic packet marking with non-preemptive compensation. IEEE Communications Letters. 8(6), 359-361 (2004)

16. A Yaar, A Perrig, D Song, in Proceedings of Symposium on Security and Privacy. Pi: a path identification mechanism to defend against DDoS attacks, (2003), pp. 93-107

17. A Yaar, A Perrig, D Song, FIT: fast Internet traceback. IEEE Computer and Communications INFOCOM. 2, 1395-1406 (2005)

18. M Goodrich, in Proceedings of the 9th ACM conference on Computer and communications security. Efficient packet marking for large-scale IP traceback, (2002), pp. 117-126

19. A Belenky, N Ansari, IP traceback with deterministic packet marking. IEEE communication letters. 7(4), 162-164 (2003)
20. A Belenky, N Ansari, On deterministic packet marking. The International Journal of Computer and Telecommunications Networking Computer Networks. 51(10), 2677-2700 (2007)

21. M Yang, RIHT: a novel hybrid ip traceback scheme. IEEE Transactions on Information Forensics and Security. 7(2), 789-797 (2012)

22. SK Rayanchu, G Barua, in Proceedings of the First international conference on Distributed Computing and Internet Technology ICDCIT. Tracing attackers with Deterministic Edge Router Marking (DERM), (2004), pp. 400-409

23. MDD Moreira, NC Fernandes, RP Laufer, OCMB Duarte, A stateless traceback technique for identifying the origin of attacks from a single packet. IEEE International Conference on Communications (ICC), 1-6 (2011)

24. THongcheng, B Jun, An incrementally deployable flow-based scheme for IP traceback. IEEE communication letters. 16(7), 1140-1143 (2012)

25. X Wang, SF Wu, J Yuill, DS Reeves, in Proceedings of the 16th international conference on Information security: Trusted information: the new decade challenge. Sleepy watermark tracing: an active network-based intrusion response framework (Paris, 11-13 June 2001), pp. 369-385

26. V Aghaei-Foroushani, N Zincir-Heywood, in The 27th IEEE International Conference on Advanced Information Networking and Applications (AINA). Deterministic and authenticated flow marking for IP traceback (Barcelona, 25-28 March 2013)

27. V Aghaei-Foroushani, N Zincir-Heywood, in IEEE International Workshop on Cyber Crime (IWCC 2013). On evaluating IP traceback schemes: a practical perspective (San Francisco, 13-24 May 2013), pp. 127-134

28. H Burch, B Cheswick, in Proceedings of the 14th USENIX Conference on System Administration LISA. Tracing anonymous packets to their approximate source, pp. 319-327

29. K Park, H Lee, On the effectiveness of probabilistic packet marking for IP traceback under denial of service attack. IEEE Computer and Communications INFOCOM. 1, 338-347 (2001)

30. D Wei, N Ansari, in Proceedings of 3rd Annual IEEE Workshop on Information Assurance, (2002). Implementing IP traceback in the Internet: an ISP perspective, pp. 326-332

31. R Shokri, H Mohammadi, N Yazdani, A Varshovi, B Sadeghian, DDPM: dynamic deterministic packet marking for IP traceback. 14th IEEE International Conference on Networks ICON. 2, 1-6 (2006)

32. R Alshammari, AN Zincir-Heywood, Can encrypted traffic be identified without port numbers, IP addresses and payload inspection? Elsevier Computer Networks. 55(6), 1326-1350 (2011)

33. DARPA 1999 intrusion detection data sets. http://www.ll.mit.edu/ mission/communications/cyber/CSTcorpora/ideval/data/. Accessed 25 October 2013

34. The CAIDA anonymized Internet traces 2012 dataset. http://www.caida. org/data/passive/passive_2012_dataset.xml. Accessed 25 October 2013

35. MAWI Working Group, MAWI (Measurement and Analysis on the WIDE Internet) traffic archive. http://mawi.wide.ad.jp/mawi/. Accessed 25 October 2013

36. A Turner, Tcpreplay suite. http://tcpreplay.synfin.net/. Accessed 25 October 2013

37. Riverbed Technology, Winpcap, The industry-standard windows packet capture library. http://www.winpcap.org/. Accessed 25 October 2013

38. D Johnson, A Menezes, S Vanstone, The Elliptic Curve Digital Signature Algorithm (ECDSA). http://cs.ucsb.edu/ koc/ccs130h/notes/ecdsa-cert. pdf. Accessed 25 October 2013

39. Agency/CentralSecurityService(NSA/CSS) National Security, The case for Elliptic Curve Cryptography. http://www.nsa.gov/business/programs/ elliptic_curve.shtml. Accessed 25 October 2013

40. Wen Dai, Crypto++ library of cryptographic schemes. http://www. cryptopp.com/. Accessed 25 October 2013

41. R Prasad, A Mihovska, New Horizons in, Mobile and Wireless Communications: Networks, Services, and Applications. (Artech House, London, 2009)

\section{doi:10.1186/1687-417X-2013-5}

Cite this article as: Aghaei-Foroushani and Zincir-Heywood: IP traceback through (authenticated) deterministic flow marking: an empirical evaluation. EURASIP Journal on Information Security 2013 2013:5. 